\title{
Fault Feature Extraction and Degradation State Identification for Piezoelectric Ceramics Cracking in Ultrasonic Motor Based on Multi-Scale Morphological Gradient
}

\author{
Guoqing An \\ Hebei University of Science and Technology, Shijiazhuang 050018, P.R. China. \\ Army Engineering University, Shijiazhuang 050003, P.R. China.
}

Hongru Li

Corresponding author, Army Engineering University, Shijiazhuang 050003, P.R. China.

\author{
Baiyan Chen \\ 63850 Troops of the PLA, Baicheng 137000, P.R. China.
}

(Received 7 December 2017; accepted 6 June 2018)

Piezoelectric ceramics cracking is one of the main faults of the ultrasonic motor. According to the morphological mathematics and information entropy, a method based on multi-scale morphological gradient was proposed for ceramics fault feature extraction and degradation state identification. To solve the problem that traditional multi-scale morphology spectral (MMS) entropy cannot exactly describe the performance degradation of the piezoelectric ceramics, multi-scale morphology gradient difference (MMGD) entropy was proposed to improve the sensitivity to the fault. Furthermore, multi-scale morphology gradient singular (MMGS) entropy was presented to reduce the system noise interference to the useful fault information. The disturbance analysis of temperature, load, and noise for MMGD entropy and MMGS entropy was also given in this paper. Combining the advantages of the above two entropies, a standard degradation mode matrix was built to distinguish the degradation state via the grey correlation analysis. The analysis of actual test samples demonstrated that this method is feasible and effective to extract the fault feature and indicate the degradation of piezoelectric cracking in ultrasonic motor.

\section{INTRODUCTION}

Ultrasonic motors have been widely used in the areas such as aerospace, medical equipment, optical instruments, robots, and new military equipment, due to some advantages of high torque, quick response, no electromagnetic, and autolocking. ${ }^{1,2}$ Piezoelectric ceramics is a key part of the ultrasonic motor. But this material is brittle, and it is prone to crack due to high frequency excitation for a long-time operation. The propagation of crack would result in the failure of the piezoelectric ceramics. It is needed to pay considerable attention to the fault feature extraction and degradation state identification for the piezoelectric ceramics cracking in ultrasonic motor. Unlike other mechanical devices, the ultrasonic motor comes with a monitor electrode used to reflect the vibration state of stator. Therefore, the monitor electrode voltage generated by the positive piezoelectric effect can be used for fault information extraction. But the rotor is driven by the frictional forces between the friction material and metal elastomer particles whose trajectory is ellipse. ${ }^{3,4}$ It seems hard to effectively extract the fault information due to the effects of other mechanical system noises. Consequently, an effective signal processing method is required.

Recently, many studies have been performed on the fault feature extraction of mechanical device. The typical methods are the wavelet transform and empirical mode decomposition (EMD). ${ }^{5}$ In the aspect of wavelet transform, Li Y. et al. used tunable Q-factor wavelet transform to separate the transient impact component related to early fault information of rolling bearing. 6 Pan et al. introduced the empirical wavelet transform to extract inherent modulation mechanical fault information by decomposing signal into mono-components. ${ }^{7}$ Singh et al. used methodology based on over-complete rational dilation wavelet transform to enable the proposed indicator be sensitive to fault severity. ${ }^{8}$ Wang D. et al. proposed Gauss-Hermite integration-based Bayesian inference method to estimate the posterior distribution of wavelet parameters, then an optimal wavelet filtering was conducted to extract bearing fault features. ${ }^{9}$ In the aspect of EMD, Xue X. et al. utilized an adaptive fast ensemble EMD to solve the problems of residue noise in the signal reconstruction and the parameters selection and extracted the fault feature of bearing with high computational ef- 
ficiency. ${ }^{10}$ Imaouchen Y. et al. tailored a frequency-weighted energy operator based on ensemble EMD and achieved very good results under the strong noise and multiple vibration interferences. ${ }^{11} \mathrm{Lv}$ Y. et al. used multivariate EMD to analyse all the frequency components related to bearing fault via the signals from different locations of machine. ${ }^{12}$ Rai A. et al. recommended a method based on EMD and k-medoids clustering to the bearing performance degradation assessment. ${ }^{13}$ Meng L. et al. propose a hybrid fault diagnosis method based on wavelet transform and EMD for the vibration signals pre-processing of defective bearings. ${ }^{14}$ But there are common problems in all the above studies: On the one hand, the selection of both thresholds and wavelet basis can hardly be solved; on the other hand, the sensitive information may not be accurately extracted by EMD because of the severe mode fixing and end effect. ${ }^{15}$

A method called morphological signal processing, which can extract fault features from strong background noise while preserving the shape characteristics of the useful components, has been studied widely in recent years. ${ }^{16}$ Zheng Z. et al. took generalized morphological fractal dimensions as the feature vectors of the gear faults. ${ }^{17}$ Yu D. et al. proposed an improved morphological component analysis method for the compound fault of the gear and the bearing in gearboxes. ${ }^{18}$ But the single-scale analysis may suffer from the completeness in the extracted features. Aiming to the above problem, Li Y. et al. presented an adaptive multi-scale morphological filter for railway wheel flat and train axle bearing fault detection. ${ }^{19,20}$

Furthermore, entropy is able to quantify the disorder or uncertainty of probability distribution, and it has been widely applied in damage monitoring or fault diagnosis. ${ }^{22} \mathrm{He}$ D. et al. took the minimum entropy deconvolution as an effective method used in fault diagnosis of vacuum pump system. ${ }^{23} \mathrm{Ai} \mathrm{Y}$. et al fused singular spectrum entropy, power spectrum entropy, wavelet space spectrum entropy and wavelet energy spectrum entropy for the rolling bearing fault diagnosis. ${ }^{24}$ Aouabdi S. et al. proposed a method for identifying gear tooth defects based on multi-scale entropy. ${ }^{25}$ However, few previous studies have applied above methods to the fault feature extraction and degradation state identification of the ultrasonic motor.

Aiming to piezoelectric ceramics cracking in ultrasonic motor, the voltage signal generated from the sensor part of stator will be used to extract the fault information. A fault feature extraction method is introduced based on multi-scale morphological decomposition and information entropy. The degradation states will be identified by grey correlation analysis. The paper is organized as follows: In Section 2, multi-scale morphological decomposition method is reviewed. Then, fault feature extraction by the multi-scale morphology gradient difference (MMGD) entropy and multi-scale morphology gradient singular (MMGS) entropy will be introduced in detail. In Section 3, combining the advantages of the MMGD entropy and MMGS entropy, a standard degeneration mode matrix will be built to distinguish the degradation states via the value of grey relevancy. The procedures for the degradation state identifica- tion processes will also be detailed. The effectiveness of the proposed method will be validated with experiment results in Section 4. Finally, the conclusions are provided in Section 5.

\section{FAULT FEATURE EXTRACTION BASED ON MULTI-SCALE MORPHOLOGY}

\subsection{Traditional Multi-scale Morphology Spectral Entropy}

In the one-dimensional signal processing based on multiscale morphology analysis, same type structural elements with different sizes are adopted to extract morphological information of the signal.

For the 1-D signal $f(n), n=0,1, \ldots, N-1$, the expansion and corrosion can be calculated by the following formula:

$$
\left\{\begin{array}{c}
(f \oplus g)(n)=\max \{f(n-m)+g(m)\} \\
(f \Theta g)(n)=\min \{f(n+m)-g(m)\}
\end{array}\right.
$$

where $g(m)$ denotes the structural element, $m=$ $0,1, \ldots, M-1, N \geq M$.

The structural element under scale $\lambda$ can be calculated by Eq. (2).

$$
\lambda g=g \oplus g \ldots \oplus g
$$

Then the expansion and corrosion calculations of signal $f(n)$ under scale $\lambda$ can be represented as follows:

$$
\left\{\begin{aligned}
(f \oplus g)_{\lambda} & =(f \oplus \lambda g)(n) \\
(f \Theta g)_{\lambda} & =(f \Theta \lambda g)(n)
\end{aligned}\right.
$$

The morphological opening and closing operation under scale $\lambda$ can be obtained by the following formula:

$$
(f \circ \lambda g)(n)=(f \Theta \lambda g \oplus \lambda g)(n)
$$

Assume that $g$ is a convex function. The traditional multi-scale morphological spectral (MMS) is shown in Eq. (5).

$$
M M S(f, \lambda, g)=-\frac{d A(f \circ \lambda g)}{d \lambda}, \lambda \geq 1 .
$$

Since $\lambda$ is continuous integer value, MMS can be calculated via the following simplified formula.

$$
M M S(\lambda, g)=A[f \circ \lambda g-f \circ(\lambda+1) g], 1 \leq \lambda \leq \lambda_{\max } ;
$$

where $A(n)=\sum_{n} f(n)$.

The normalized MMS entropy is described by Eq. (7). ${ }^{26}$

$$
E_{M M S}=\frac{-\sum_{\lambda=1}^{\lambda_{\max }} q(\lambda) \ln q(\lambda)}{\ln \lambda_{\max }} ;
$$

where $q(\lambda)=\frac{\operatorname{MMS}(\lambda, g)}{\sum M M S(\lambda, g)}$. 


\subsection{MMGD Entropy}

The morphological open operation is the key of MMS entropy. But this operator is usually accompanied by statistical bias. ${ }^{27}$ Gradient is often used as an edge detection tool in signal processing. When the travelling wave passes through the crack, the wave transmission would be subjected to a disturbance. Based on the above analysis, the concept of gradient can be introduced into the fault feature extraction of piezoelectric ceramics.

The gradient under scale $\lambda$ is defined as follows:

$$
\operatorname{Grad}(f)=(f \oplus g)_{\lambda}-(f \Theta g)_{\lambda} .
$$

MMGD is shown in Eq. (9).

$$
M M G D(f, \lambda, g)=-\frac{d A(f \oplus \lambda g-f \Theta \lambda g)}{d \lambda}, \lambda \geq 1 .
$$

Since $\lambda$ is continuous integer value, MMGD can be calculated via the following simplified formula.

$$
\begin{array}{r}
M M G D(\lambda, g)=A[(f \oplus(\lambda+1) g-f \Theta(\lambda+1) g) \\
-(f \oplus \lambda g-f \Theta \lambda g)], 1 \leq \lambda \leq \lambda_{\max }
\end{array}
$$

where $A(n)=\sum_{n} f(n)$.

The normalized MMGD entropy is described by Eq. (11).

$$
E_{M M G D}=\frac{-\sum_{\lambda=1}^{\lambda_{\max }} q(\lambda) \ln q(\lambda)}{\ln \lambda_{\max }} ;
$$

where $q(\lambda)=\frac{M M G D(\lambda, g)}{\sum M M G D(\lambda, g)}$.

MMGD entropy will be taken as one fault feature for degradation state identification of the piezoelectric ceramics cracking in this paper.

\subsection{MMGS Entropy}

As the motor load increases, the amplitude of stator vibration and the voltage generated from by the sensor part of piezoelectric ceramics will decrease. It often leads to attenuation of fault information in the signal and brings difficulty for fault feature extraction due to noise interference. Singular value decomposition (SVD) can effectively realize the separation of weak fault information and achieve good results in fault diagnosis. $^{28,29}$ MMGS entropy is proposed based on morphological gradient and SVD, and the calculating procedures are detailed as follows:

1. According to Eq. (8), calculate gradients components under different $\lambda$, and noted as $d_{\lambda}\left(\lambda=1,2, \ldots, \lambda_{\max }\right)$;

2. Construct initial matrix $A=\left[d_{1}, d_{2}, \ldots, d_{\lambda_{\max }}\right]^{T}$. Calculate the singular values noted as $\sigma_{1}^{2}, \sigma_{2}^{2}, \ldots, \sigma_{\lambda_{\max }}^{2}$;

3. Define MMGS entropy:

$$
E_{M M G S}=\frac{-\sum_{\lambda=1}^{\lambda_{\max }} q(\lambda) \ln q(\lambda)}{\ln \lambda_{\max }}
$$

where $q(\lambda)=\frac{\sigma_{\lambda}^{2}}{\sigma_{1}^{2}+\sigma_{2}^{2}+\cdots+\sigma_{\lambda \max }^{2}}$.
MMGS entropy will be taken as another fault feature for degradation state identification of the piezoelectric ceramics cracking.

\section{DEGRADATION STATE IDENTIFICATION BASED ON GREY RELATIONAL ANALYSIS}

The degradation states are divided into four categories: normal state, slight degradation, severe degradation, and failure state. The original signal generated from the sensor part of the piezoelectric ceramics is used for fault feature extraction. Procedures for the degradation state identification processes based on grey relational analysis are detailed in the following:

Step 1. Constructing a standard degeneration mode matrix

Select standard degradation data collected in normal state, slight degradation, severe degradation, and failure state under a working condition. Calculate the MMGD entropy and MMGS entropy values of four standard states to construct a standard degeneration mode matrix as follows:

$$
E=\left[\begin{array}{cc}
E_{M M G D \_n o r m a l} & E_{M M G S \_n o r m a l} \\
E_{M M G D \_s l i g h t} & E_{M M G S \_s l i g h t} \\
E_{M M G D \_s e v e r e} & E_{M M G S \_s e v e r e} \\
E_{M M G D \_f a i l u r e} & E_{M M G S \_f a i l u r e}
\end{array}\right] .
$$

Step 2. Calculating of grey relevance between test sample and standard matrix

The sequence based on MMGD entropy and MMGS entropy of test sample is noted as follows:

$$
\begin{array}{r}
e_{0}=\left\{e_{0}(k) \mid k=1,2\right\}=\left(e_{0}(1), e_{0}(2)\right) \\
=\left(E_{M M G D \_t e s t}, E_{M M G S \_t e s t}\right) ;
\end{array}
$$

And the sequence of elements in standard degeneration mode matrix is shown below.

$$
e_{i}=\left\{e_{i}(k) \mid k=1,2\right\}=\left(e_{0}(1), e_{0}(2)\right), i=1,2, \cdots, 4 ;
$$

Then the value of grey relevance $e_{i}$ to $e_{0}$ is

$$
r_{i}=\frac{1}{2} \sum_{k=1}^{2} \xi_{i}(k)
$$

where

$$
\begin{array}{r}
\xi_{i}(k)= \\
\frac{\rho \cdot \max _{i} \max _{k}\left|e_{0}(k)-e_{i}(k)\right|}{\left|e_{0}(k)-e_{i}(k)\right|+\rho \cdot \max _{i} \max _{k}\left|e_{0}(k)-e_{i}(k)\right|}, \\
\rho \in(0,1) ;
\end{array}
$$

$\rho$ denotes the distinguishing coefficient. The smaller the value of $\rho$, the better the distinguish ability.

Step 3. Identification of degradation state

The state corresponding to the maximum value of grey relevance, is seen as the result of degradation state identification.

The flowchart of identification is shown in Fig. 1. 


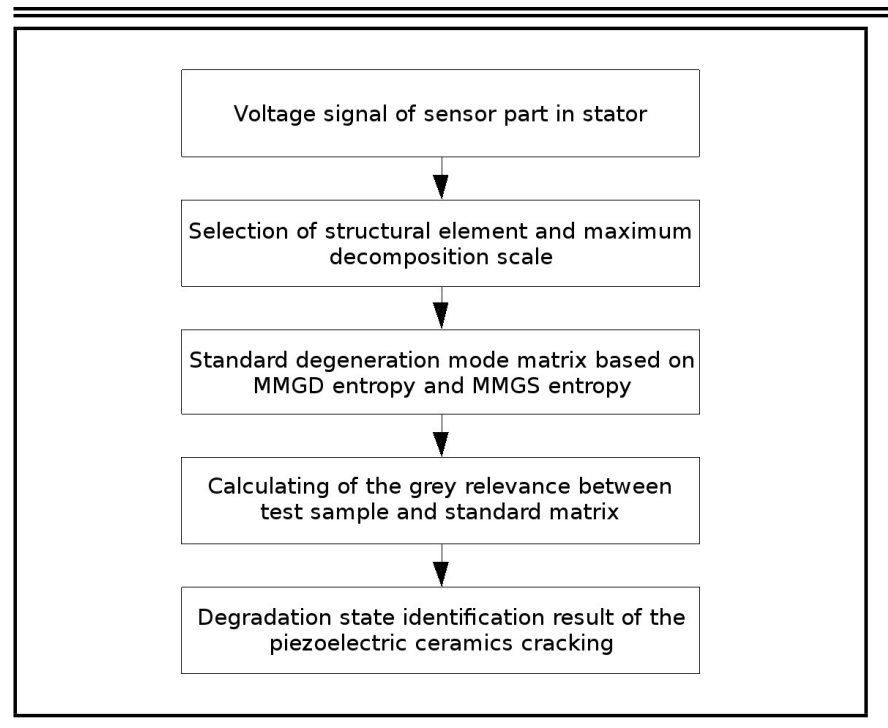

Figure 1. The flowchart of identification.

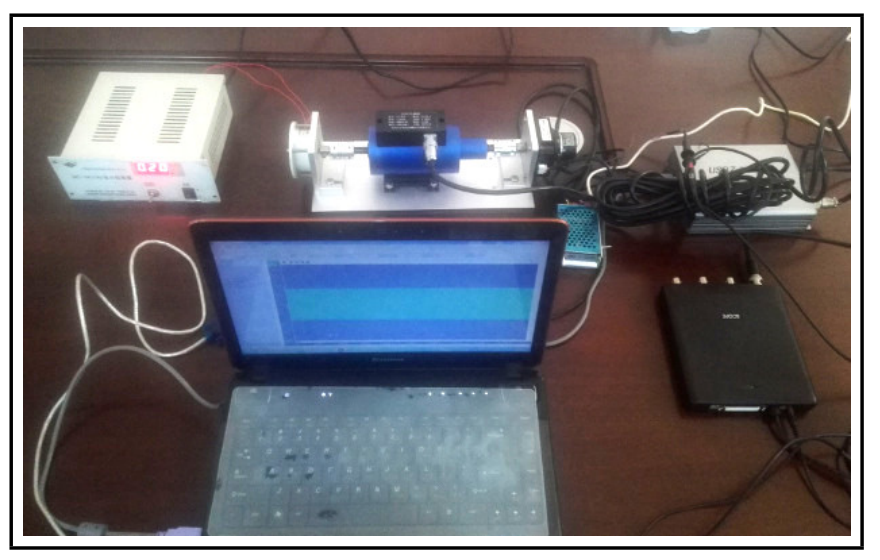

Figure 2. Test-bed for ultrasonic motor.

\section{EXPERIMENTAL RESULTS}

\subsection{Sampling of Degradation Data}

The sampling of ceramics cracking degradation data was carried out by the ultrasonic motor test-bed, which is shown in Fig. 2.

The tested ultrasonic motor is TRUM-60-P. Its maximum torque is $0.6 \mathrm{~N} \cdot \mathrm{m}$, and no-load speed is $120 \mathrm{rpm}^{-1}$. The speed is controlled by a variable frequency driver, and the load can be changed by varying the exciting current of a magnetic powder brake. The three load levels are respectively $0 \mathrm{~N} \cdot \mathrm{m}, 0.1 \mathrm{~N} \cdot \mathrm{m}$, and $0.2 \mathrm{~N} \cdot \mathrm{m}$. The signal frequency generated from the sensor part of the stator is $40.65 \mathrm{kHz}$, and the signal is sampled and stored by the Handyscope HS4 data acquisition card of TiePie engineering. The sampling frequency is set to be $500 \mathrm{kHz}$, and the sampling time of each group data is $2 \mathrm{~s}$. There are 50 groups of data that were collected under each load level. The piezoelectric ceramics of four degradation states are shown in Fig. 3.

Taking the $0 \mathrm{~N} \cdot \mathrm{m}$ load as an example, the waveform of the voltage signal generated from the sensor part is shown as Fig. 4.

From Fig. 4, it can be seen that the envelope changes of

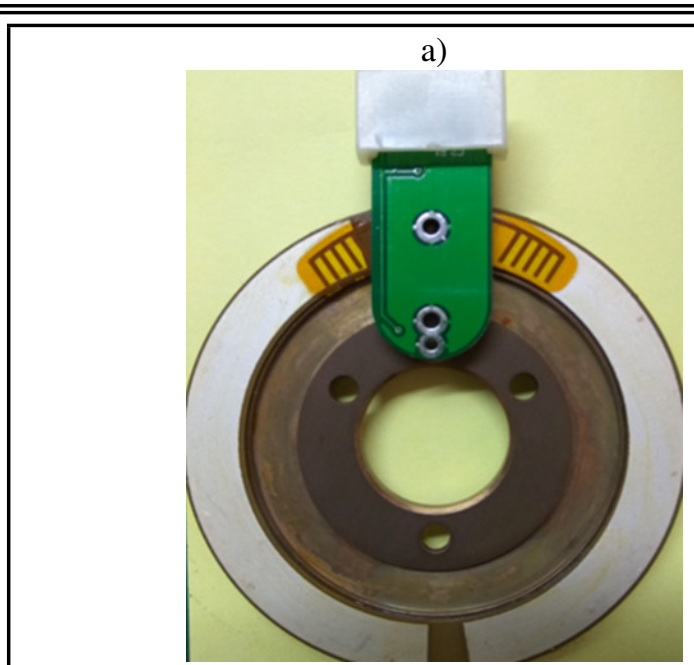

b)

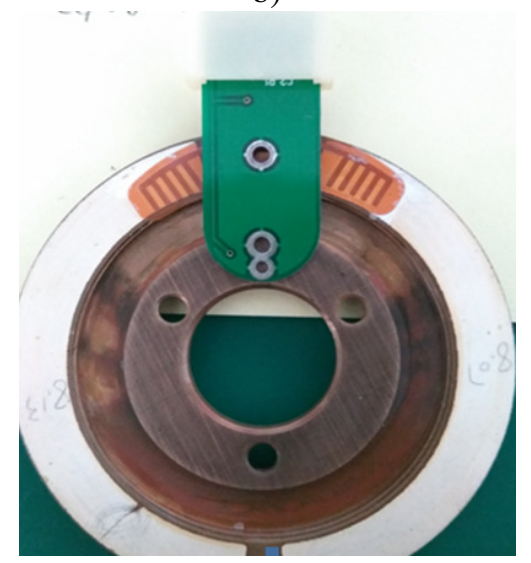

c)

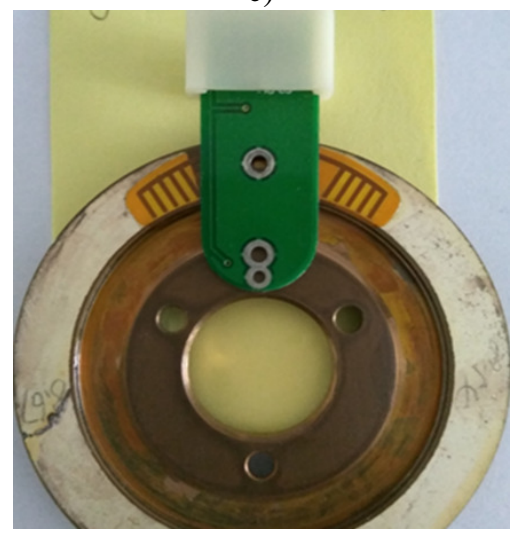

d)

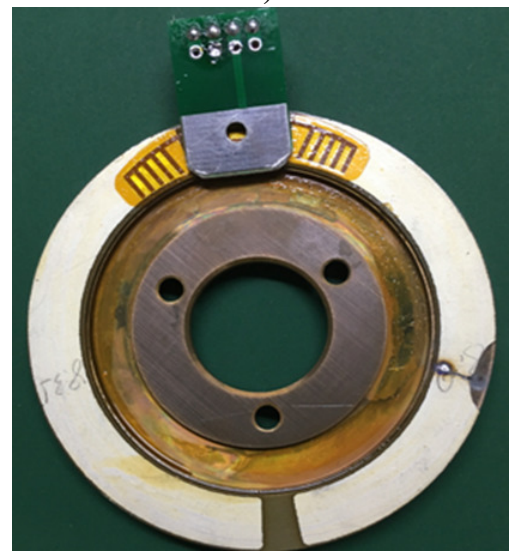

Figure 3. Piezoelectric ceramics of four measured motors with different degradation states. 


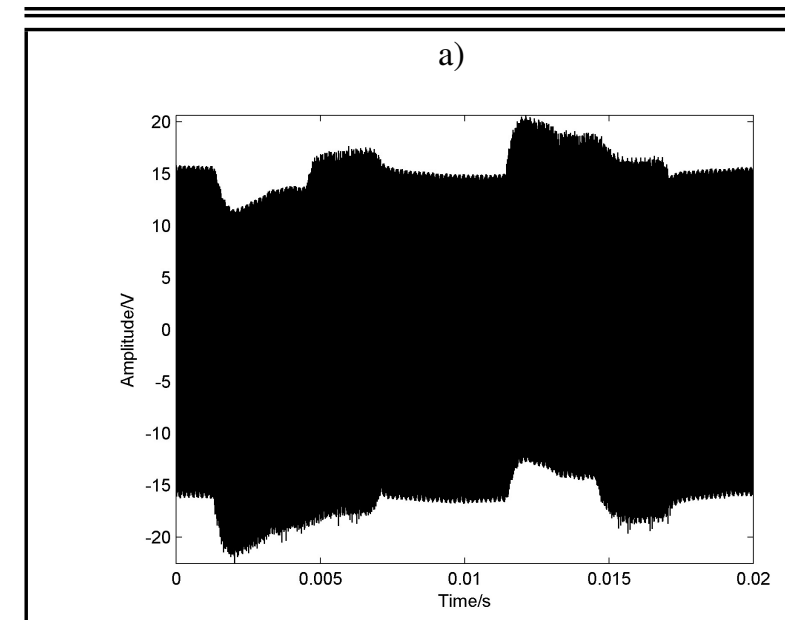

b)

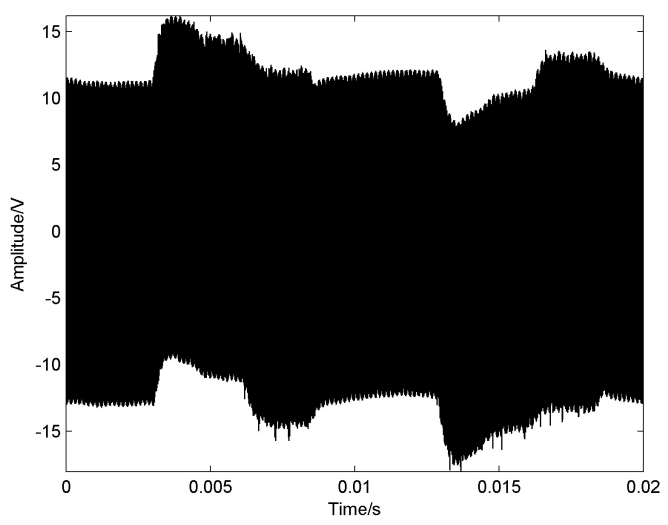

c)

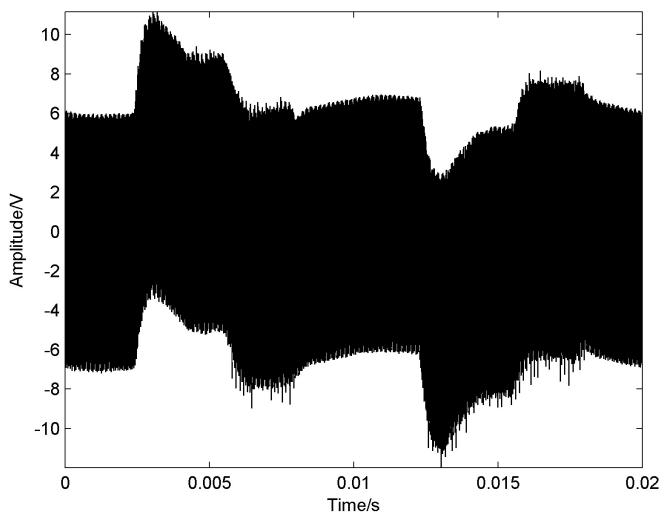

d)

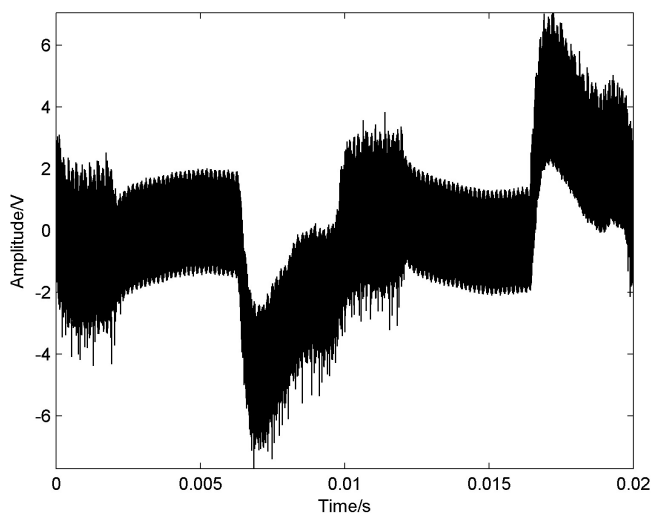

Figure 4. The waveform of the data under $0 \mathrm{~N} \cdot \mathrm{m}$ load. a)

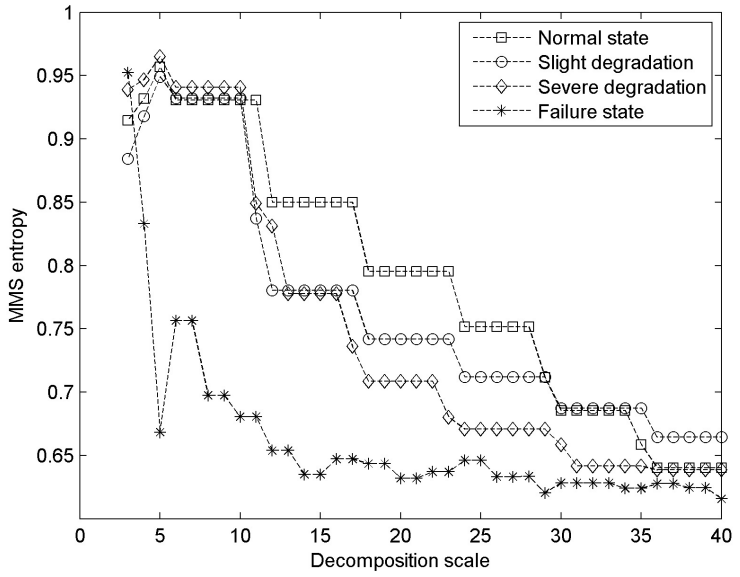

b)

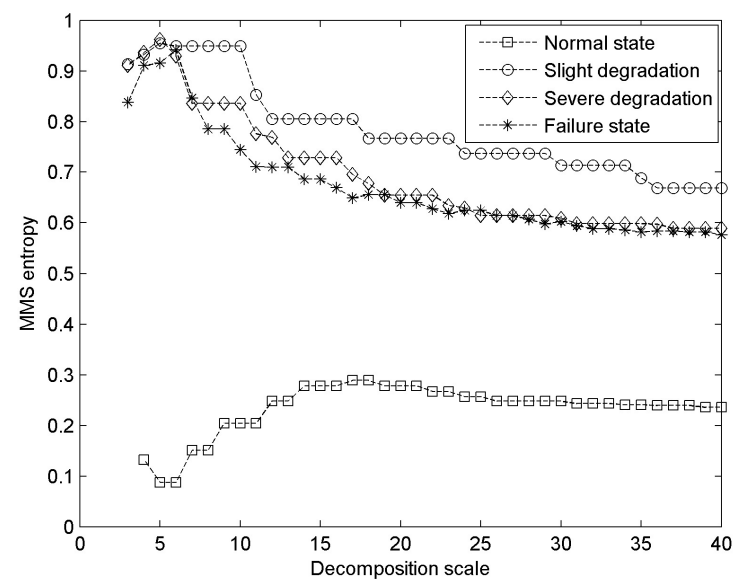

c)

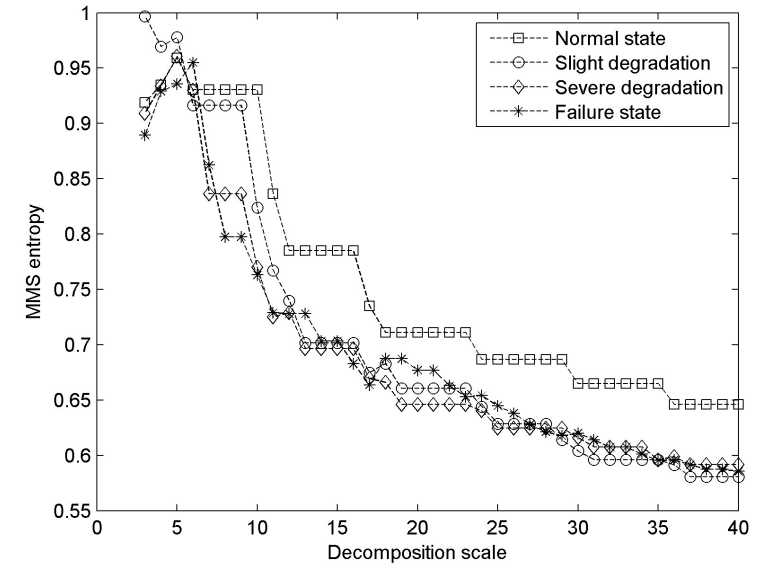

Figure 5. Calculating results of MMS entropy in different scale.

the signals become more and more violent with the development of degradation, but the time domain characteristics are not particularly evident, especially among the first three degradation states. Time domain analysis cannot be used to achieve the degradation state identification. Therefore, the proposed entropy based on multi-scale morphological gradient will be used to extract the degradation feature in this paper. 


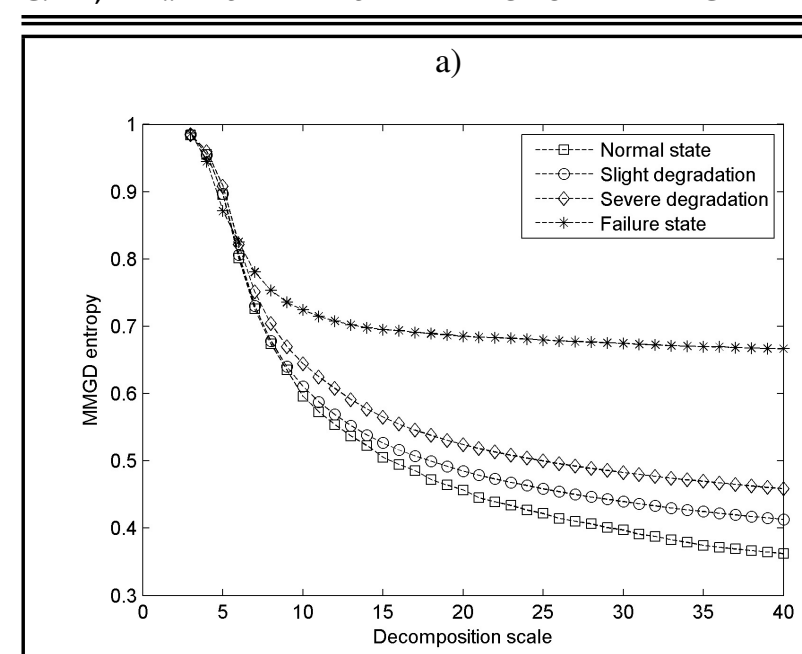

b)

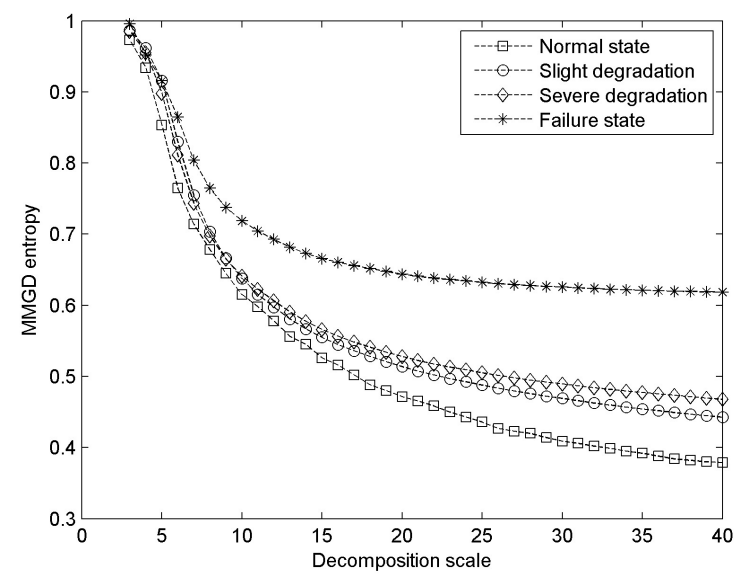

c)

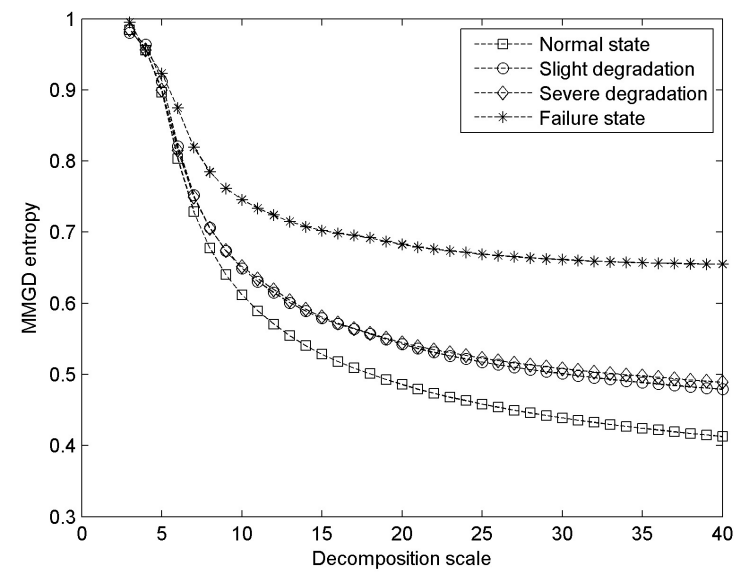

Figure 6. Calculating results of MMGD entropy in different scale.

\subsection{Selection of Decomposition Scale}

One group of standard degradation data in each state of normal, slight, severe, and failure was selected. The flat structure element $g=[0,0,0]$ is used for multi-scale morphological analysis, and the largest scale is 40 .

The calculating results of MMS entropy in different scale are shown in Fig. 5.

It can be concluded that, the result reflects a wrong logical relationship under the load of $0.1 \mathrm{~N} \cdot \mathrm{m}$. Under the load a)

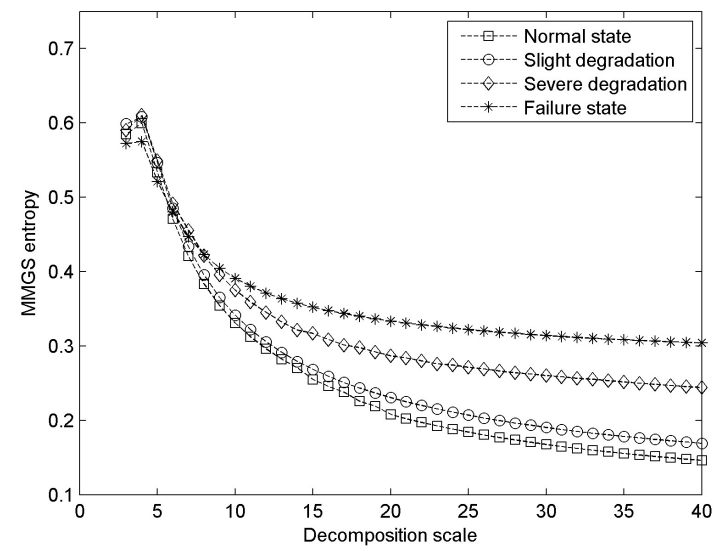

b)

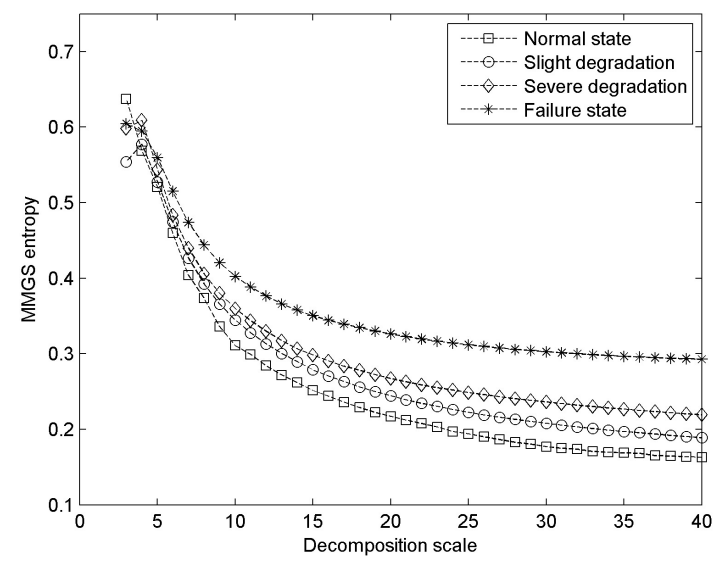

c)

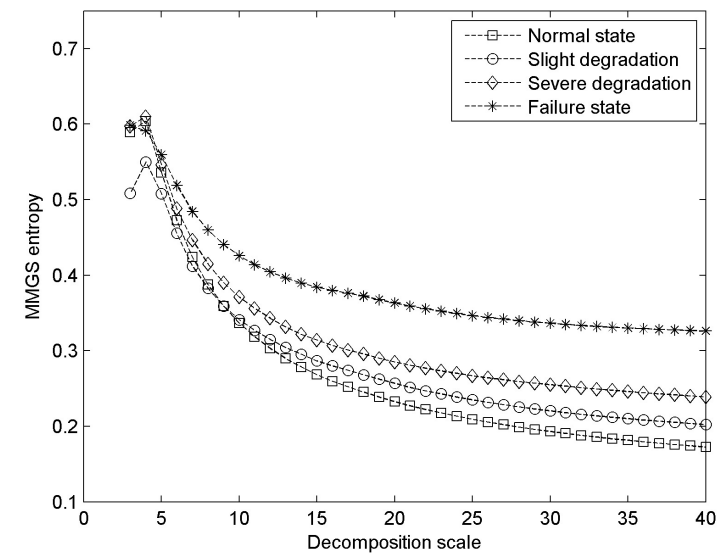

Figure 7. Calculating results of MMGS entropy in different scale.

of $0.2 \mathrm{~N} \cdot \mathrm{m}$, there are three curves intertwined to present a bad degradation states distinction. The main reason is that, the open operation is usually accompanied by statistical bias. MMS entropy cannot be taken as the fault feature because the correctness cannot be guaranteed.

The calculating results of MMGD entropy and MMGS entropy in different scale are shown in Fig. 6 and Fig. 7.

From Fig. 6 and Fig. 7, it can be found that both of MMGD entropy and MMGS entropy can reflect a reasonable logical relationship in all above load conditions. The entropy values 


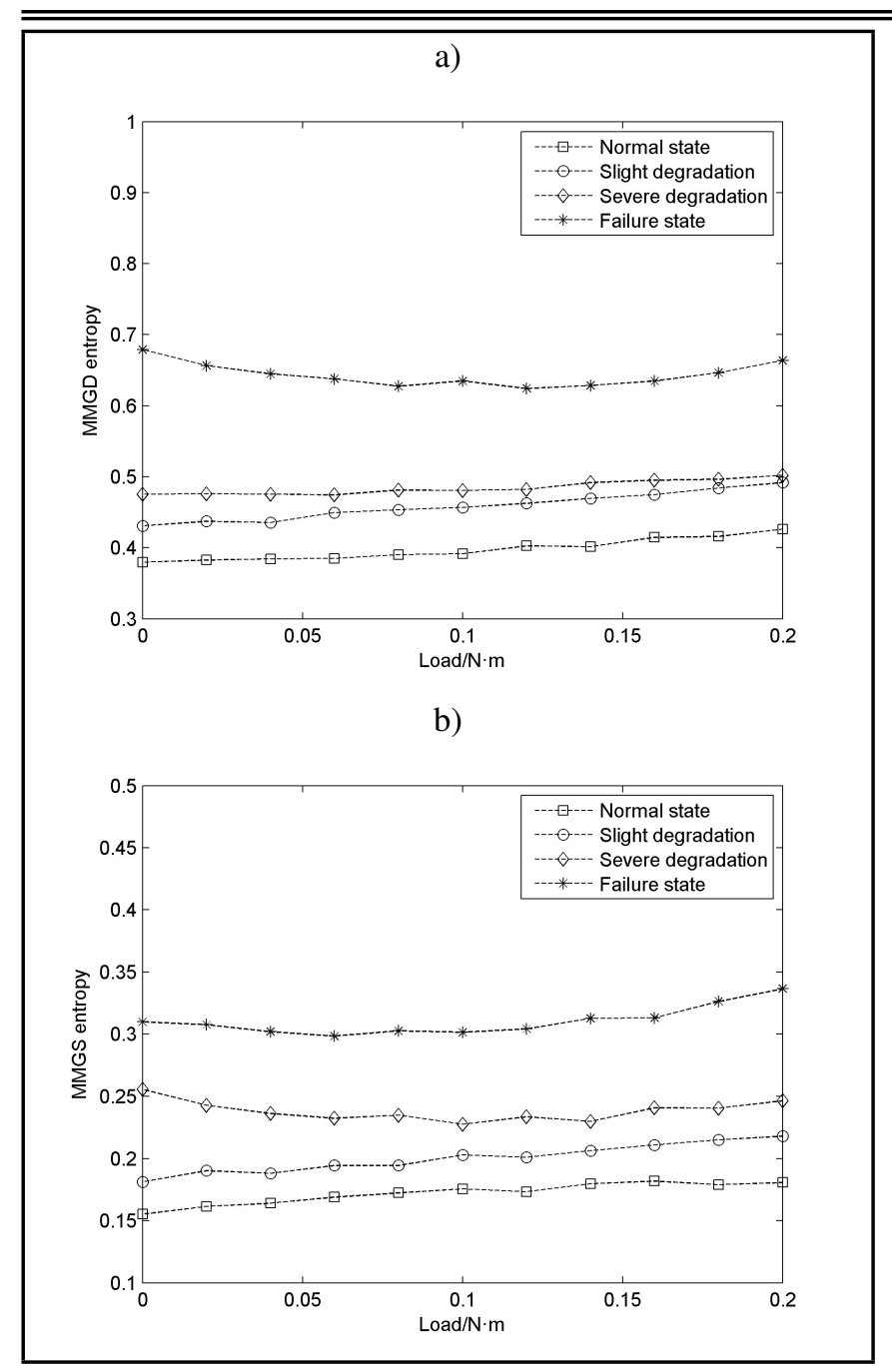

Figure 8. The changes of MMGD and MMGS entropy along with the load.

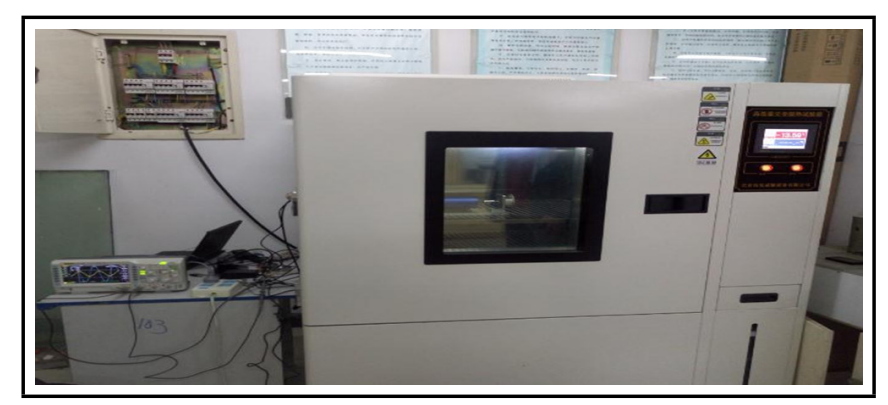

Figure 9. Thermostat box.

under the same scale increase with the state deterioration. So MMGD entropy and MMGS entropy can be used as the fault feature to reflect the degradation state of piezoelectric ceramics cracking. The bigger the absolute value of certain scale, it will be more sensitive to identify the performance degradation of piezoelectric ceramics. ${ }^{30}$ According to the discrimination of curves and the calculation cost in Fig. 6 and Fig. 7, the maximum scale of MMGD entropy and MMGS entropy is selected as 36 .

\subsection{Disturbance Analysis}

The disturbances in the actual operation of an ultrasonic motor mainly include three aspects: load, ambient temperature, a)

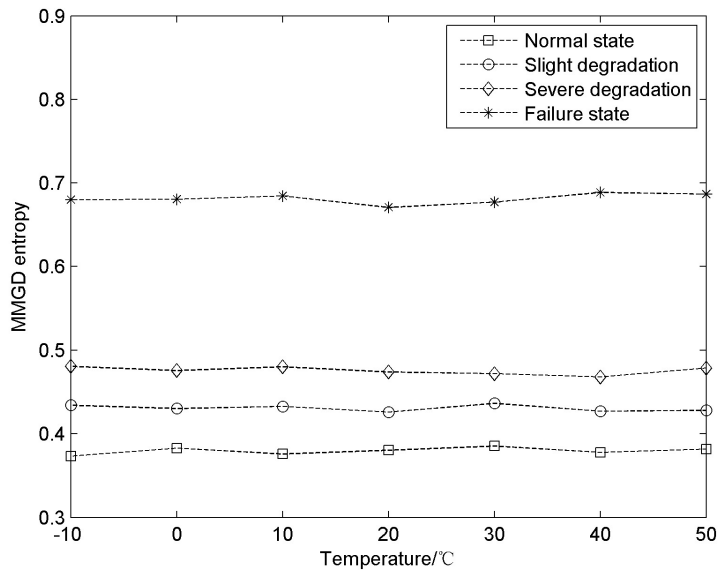

b)

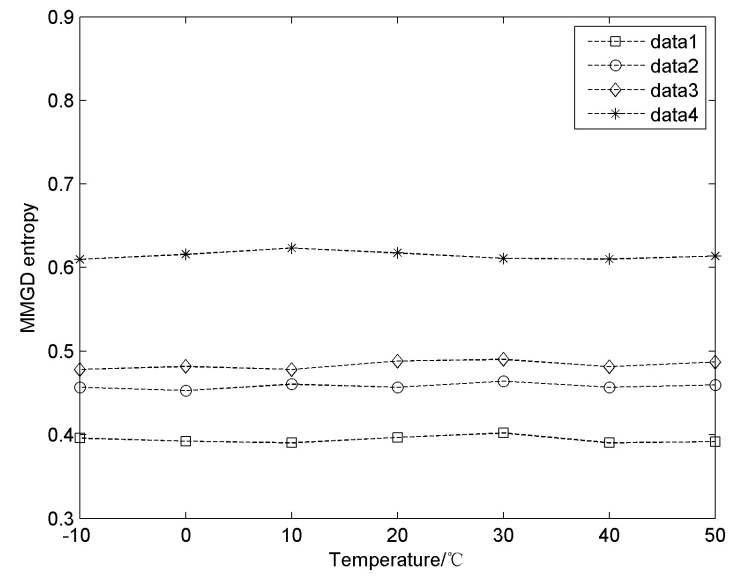

c)

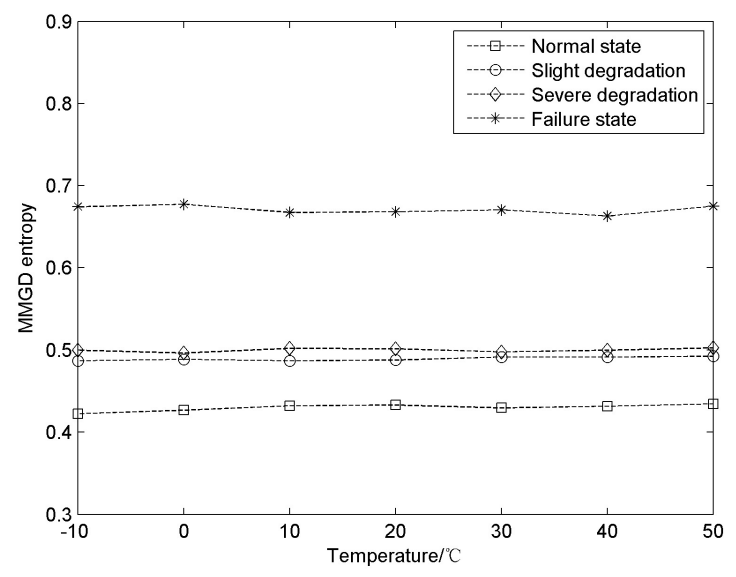

Figure 10. Calculating results of MMGD entropy in different ambient temperature.

and system noise. The disturbance analysis of MMGD entropy and MMGS entropy is discussed as following:

\subsubsection{Load change}

The mechanical characteristic of ultrasonic motor is relatively soft, and the speed of motor would fluctuate due to the load variation on the shaft. ${ }^{31}$ The disturbance analysis of the load changes is necessary. Adjust the load at $0.02 \mathrm{~N} \cdot \mathrm{m}$ inter- 
a)

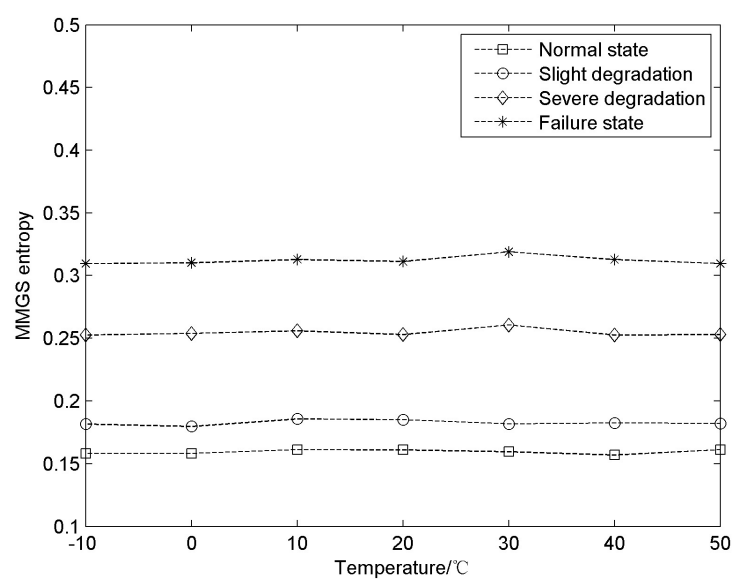

b)

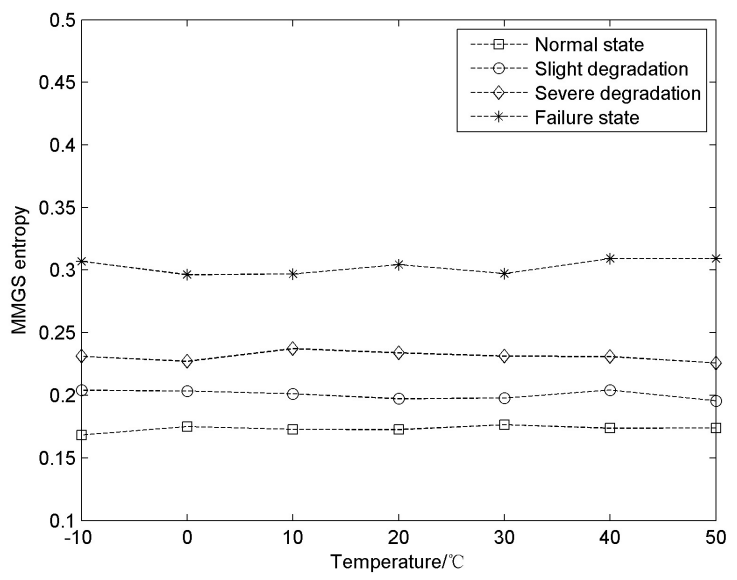

c)

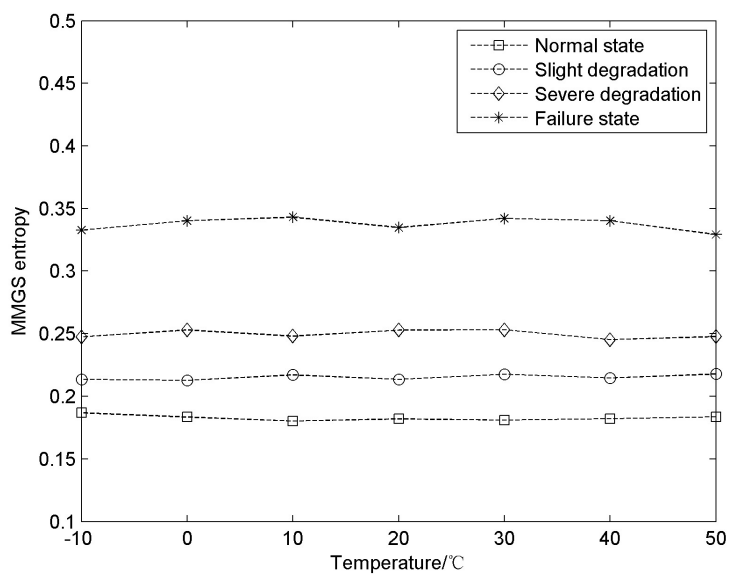

Figure 11. Calculating results of MMGS entropy in different ambient temperature.

vals in the range of $0.2 \mathrm{~N} \cdot \mathrm{m}$. Select one group of data at each load level. The changes of MMGD entropy and MMGS entropy $(\lambda=36)$ along with the load are shown in Fig. 8.

The above figure reflects the degradation indication ability of MMGD entropy and MMGS entropy with different loads. We conclude that the two entropies can keep clear uptrend as the fault degradation. For MMGD entropy, the difference between slight and severe degradation decreases gradually, and the two curves almost coincide especially under the load of $0.2 \mathrm{~N} \cdot \mathrm{m}$. Compared with MMGD entropy, MMGS entropy has better discrimination with the heavier load, but it is worth noting that MMGD entropy has a better ability to identify the early fault due to bigger absolute value between normal state and slight degradation.

\subsubsection{Ambient temperature change}

The change of ambient temperature will cause the drift of motor impedance characteristics, and the resonance frequency ultrasonic motor will also change. ${ }^{32}$ Once the driving frequency could not track this change, it would result in the decrease of the speed and efficiency. In order to study the effect of ambient temperature on the two entropies, a thermostat box (GDJS-408C) is selected for the experiment, which is shown in Fig. 9.

The temperature was adjusted at $10^{\circ}$ intervals from $-10^{\circ}$ to $50^{\circ}$ (the suggested ambient temperature range for the measured ultrasonic motor), and one group of data at each temperature observation point was selected. The changes in MMGD entropy and MMGS entropy $(\lambda=36)$ are shown in Fig. 10 and Fig. 11.

From Fig. 10 and Fig. 11, we conclude that the ambient temperature has little effect on the MMGD entropy and MMGS entropy. The two entropies keep gradually increasing with the development of degradation in the suggested ambient temperature range. The main reason is that, the change of ambient temperature would cause the drift of motor impedance characteristics, but it does not lead to the change of mathematical morphology of signal. Therefore, the two entropies which are based on morphological gradient have good anti-interference performance to the ambient temperature.

\subsubsection{Noise interference}

In order to study the adaptability of the MMGD entropy and MMGS entropy to vibration noise environment, several different intensities of simulation noise are added to the original measured signals for further analysis. In this paper, five kinds of signal-to-noise ratio (SNR) are set up respectively: $0 \mathrm{~dB}$, $5 \mathrm{~dB}, 10 \mathrm{~dB}, 15 \mathrm{~dB}$, and $20 \mathrm{~dB}$. The SNR of the original measured signal is marked as $\infty$. The waveform of the simulation signal with $0 \mathrm{~dB}$ noise is shown as Fig. 12.

The calculating results of MMGD entropy and MMGS entropy with different intensity noise are shown in Fig. 13 and Fig. 14.

The above figures show that two entropies increase with noise intensity as a whole. Both of the fault features keep gradually increasing with the development of degradation no matter what the noise background is. But it is also clear that the increase scope of MMGD entropy is narrowing as the noise is strengthened, while the performance of MMGS entropy is less affected by the added noise. The reason is analysed and concluded as SVD included in the MMGS entropy takes effect in the separation of weak fault information. ${ }^{33,34}$ Therefore, com- 
a)

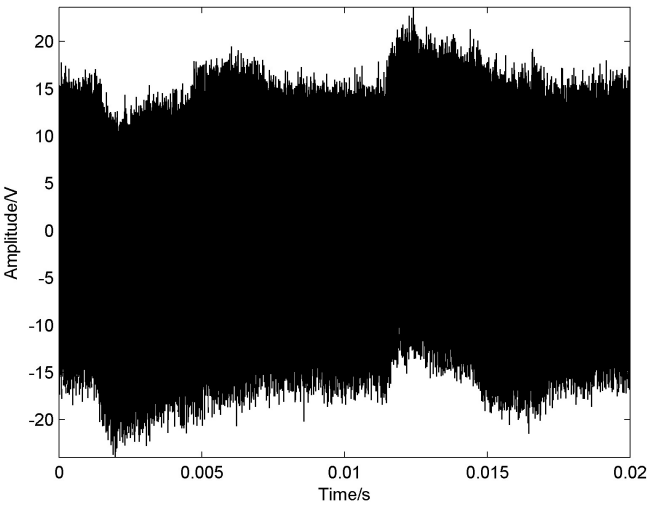

b)

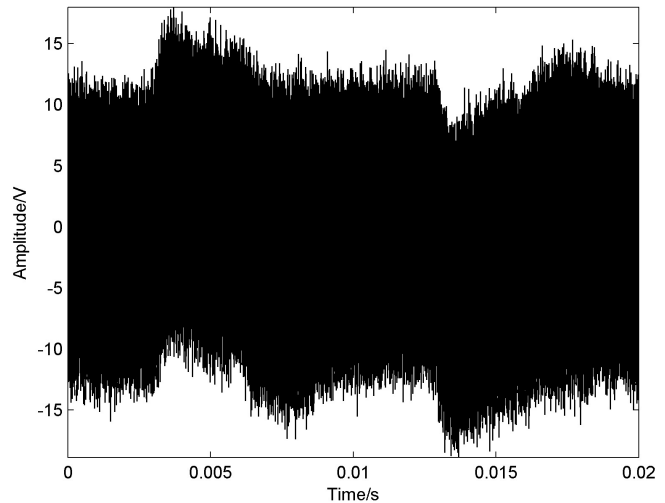

c)

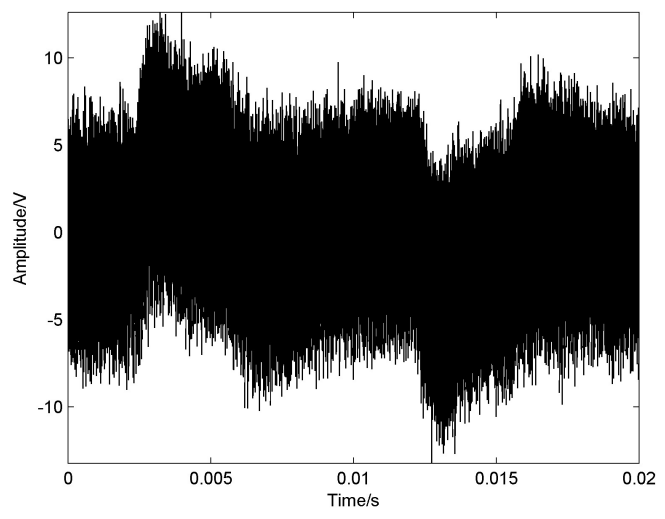

d)

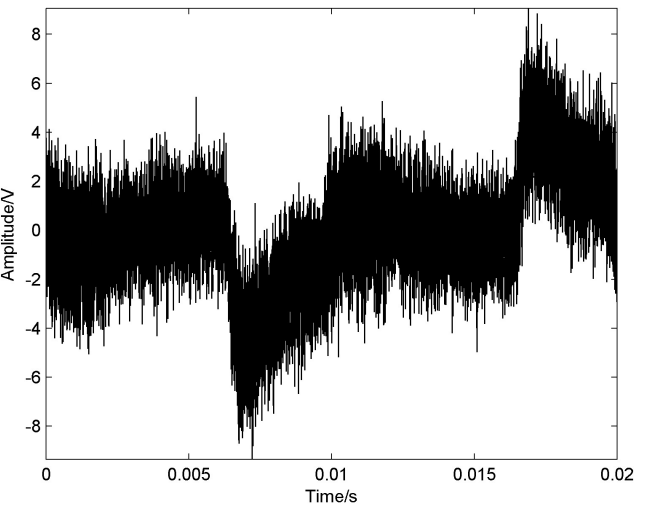

Figure 12. The waveform of the voltage with noise. a)

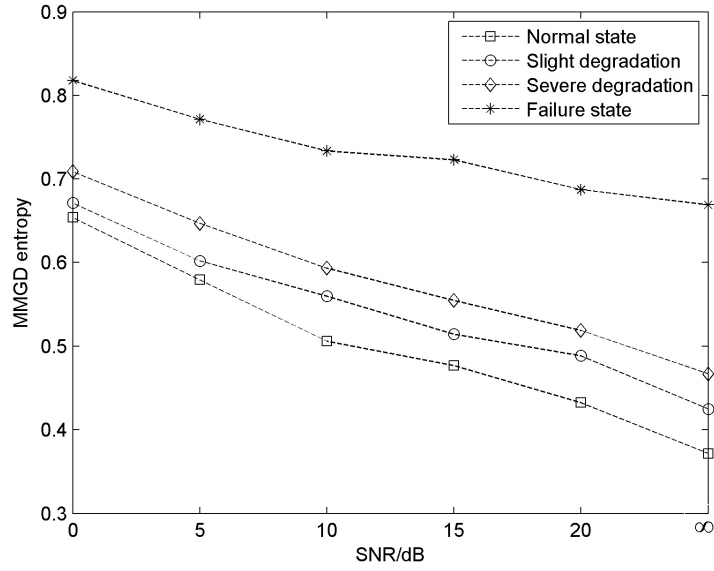

b)

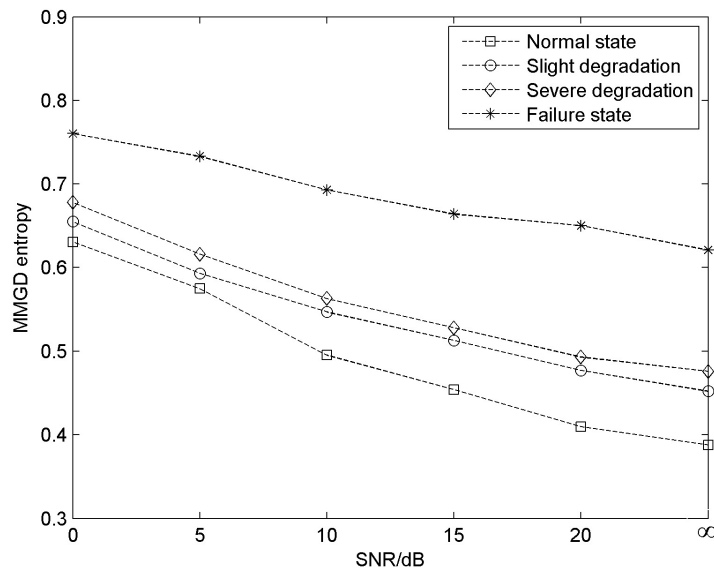

c)

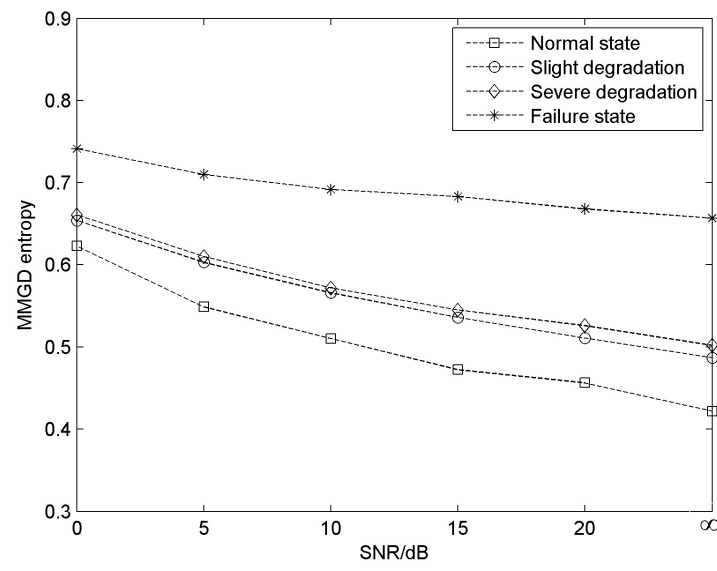

Figure 13. Calculating results of MMGD entropy with different intensity noise.

pared with MMGD entropy, MMGS entropy has better antinoise performance.

\subsection{Fault Feature Extraction And Degradation State Identification}

In 50 groups of collected data from each degradation state, 10 groups of data are selected as training samples to construct the standard degeneration mode matrixes. The selected 


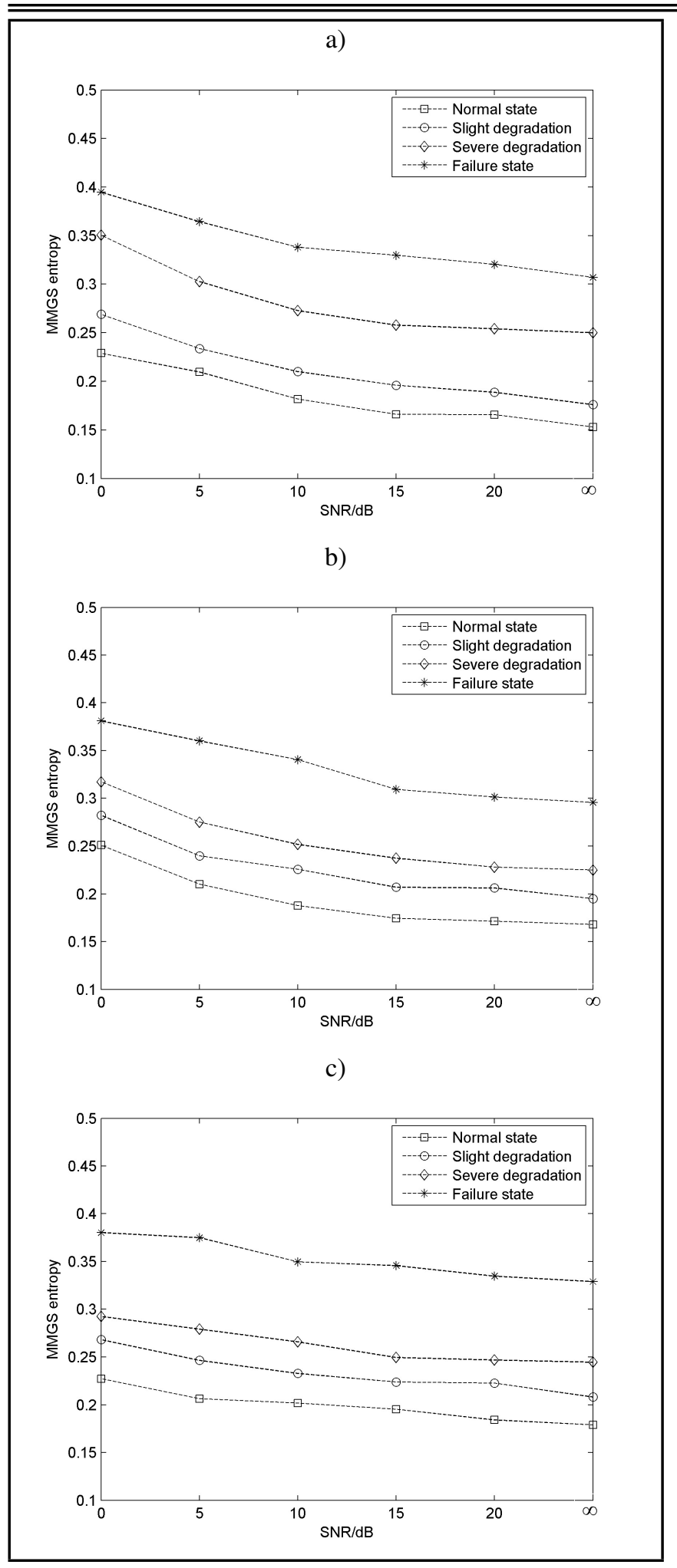

Figure 14. Calculating results of MMGS entropy with different intensity noise.

10 group numbers are $3,8,13,18,23,28,33,38,43$, and 48. The remaining 40 groups of collected data are used as test samples. So, there are 160 sets of test samples in 4 degenerate states, where the 0-40 groups are normal state data, the 41-80 groups are slight degradation data, the $81-120$ groups are severe degradation data, and the 121-160 groups are failure state data. In the grey correlation analysis, the resolution ratio $\rho$ is selected as 0.5 . a)

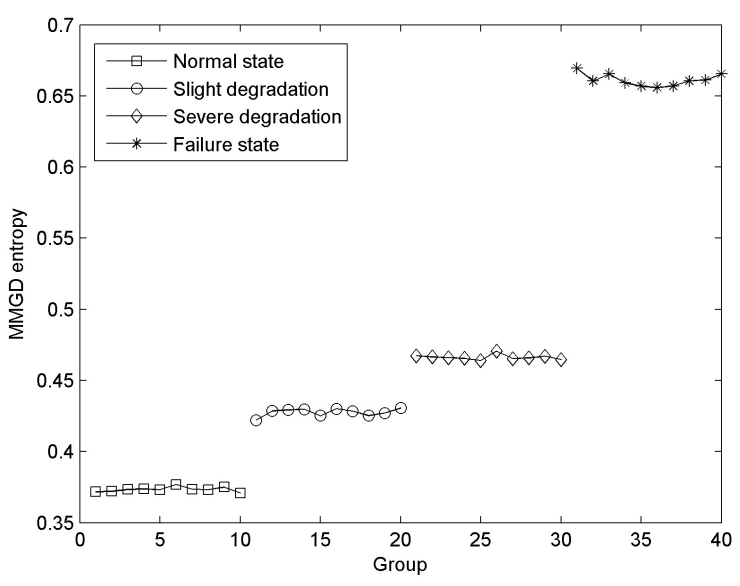

b)

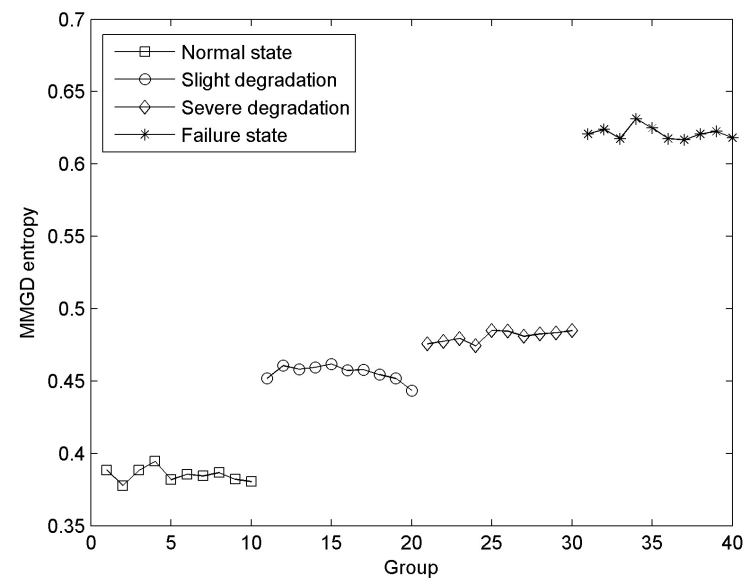

c)

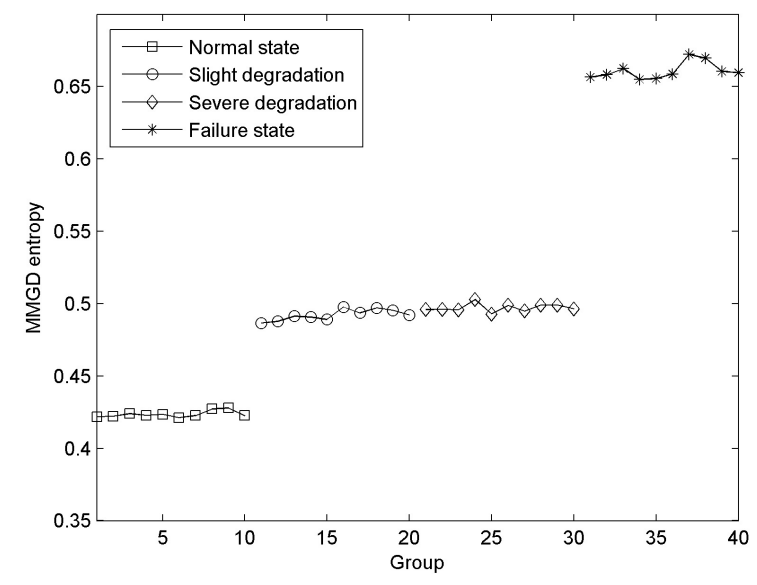

Figure 15. MMGD entropy values of training samples.

\subsubsection{Degradation state identification based on MMGD entropy}

The MMGD entropy values of 10 training samples under maximum scale 36 are shown in Fig. 15.

Calculate the average MMGD entropy values of 10 training samples in different degradation states, and then the constructed standard degeneration mode vectors $E_{0}, E_{1}, E_{2}$ in 
Table 1. Statistical results based on MMGD entropy.

\begin{tabular}{||c|c|c|c|c||}
\hline Load & $\begin{array}{c}\text { Test } \\
\text { sample }\end{array}$ & $\begin{array}{c}\text { Wrong } \\
\text { identification }\end{array}$ & Load & $\begin{array}{c}\text { Total } \\
\text { identification ratio }\end{array}$ \\
\hline $0 \mathrm{~N} \cdot \mathrm{m}$ & 160 & 7 & $95.63 \%$ & \\
$0.1 \mathrm{~N} \cdot \mathrm{m}$ & 160 & 11 & $93.11 \%$ & $90.63 \%$ \\
$0.2 \mathrm{~N} \cdot \mathrm{m}$ & 160 & 27 & $83.13 \%$ & \\
\hline
\end{tabular}

three load levels are defined as follows.

$$
\begin{aligned}
& E_{0}=\left[\begin{array}{l}
0.373 \\
0.428 \\
0.466 \\
0.661
\end{array}\right] ; \\
& E_{1}=\left[\begin{array}{l}
0.385 \\
0.456 \\
0.481 \\
0.621
\end{array}\right] ; \\
& E_{2}=\left[\begin{array}{l}
0.424 \\
0.492 \\
0.497 \\
0.661
\end{array}\right] .
\end{aligned}
$$

According to the value of grey relevance between test samples and standard vector, the degradation state recognition results are shown in Fig. 16 and Tab. 1.

From Fig. 16 and Tab. 1, it can be found that the identification ratio of the method based on MMGD entropy is gradually reduced with the increase of load. Therefore, MMGD entropy is influenced by the load of the motor, but it shows a high identification ratio of normal state and slight degradation.

\subsubsection{Degradation state identification based on MMGS entropy}

The MMGS entropy values of training samples under maximum scale 36 are shown in Fig. 17.

Calculate the average MMGS entropy values of 10 training samples in different degradation states, and then the constructed standard degeneration mode vectors $E_{0}, E_{1}, E_{2}$ in three load levels are defined as follows:

$$
\begin{aligned}
& E_{0}=\left[\begin{array}{l}
0.155 \\
0.173 \\
0.248 \\
0.302
\end{array}\right] ; \\
& E_{1}=\left[\begin{array}{l}
0.167 \\
0.196 \\
0.230 \\
0.298
\end{array}\right] ; \\
& E_{2}=\left[\begin{array}{l}
0.180 \\
0.204 \\
0.243 \\
0.332
\end{array}\right] .
\end{aligned}
$$

a)

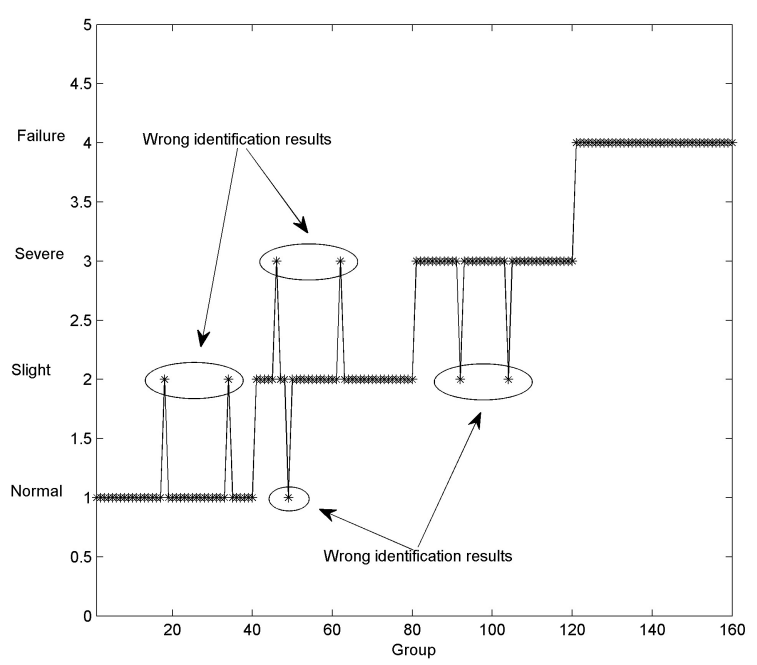

b)

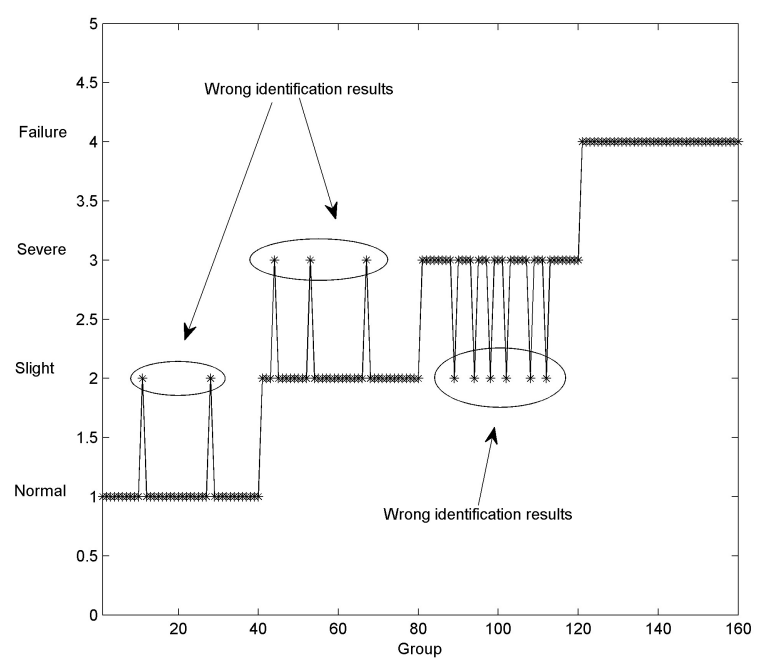

c)

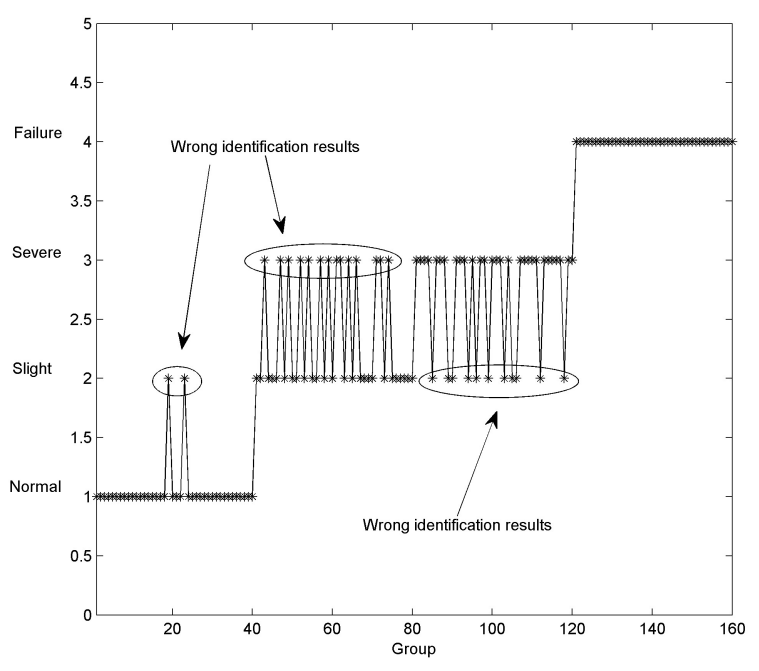

Figure 16. Degradation state identification results based on MMGD entropy. 


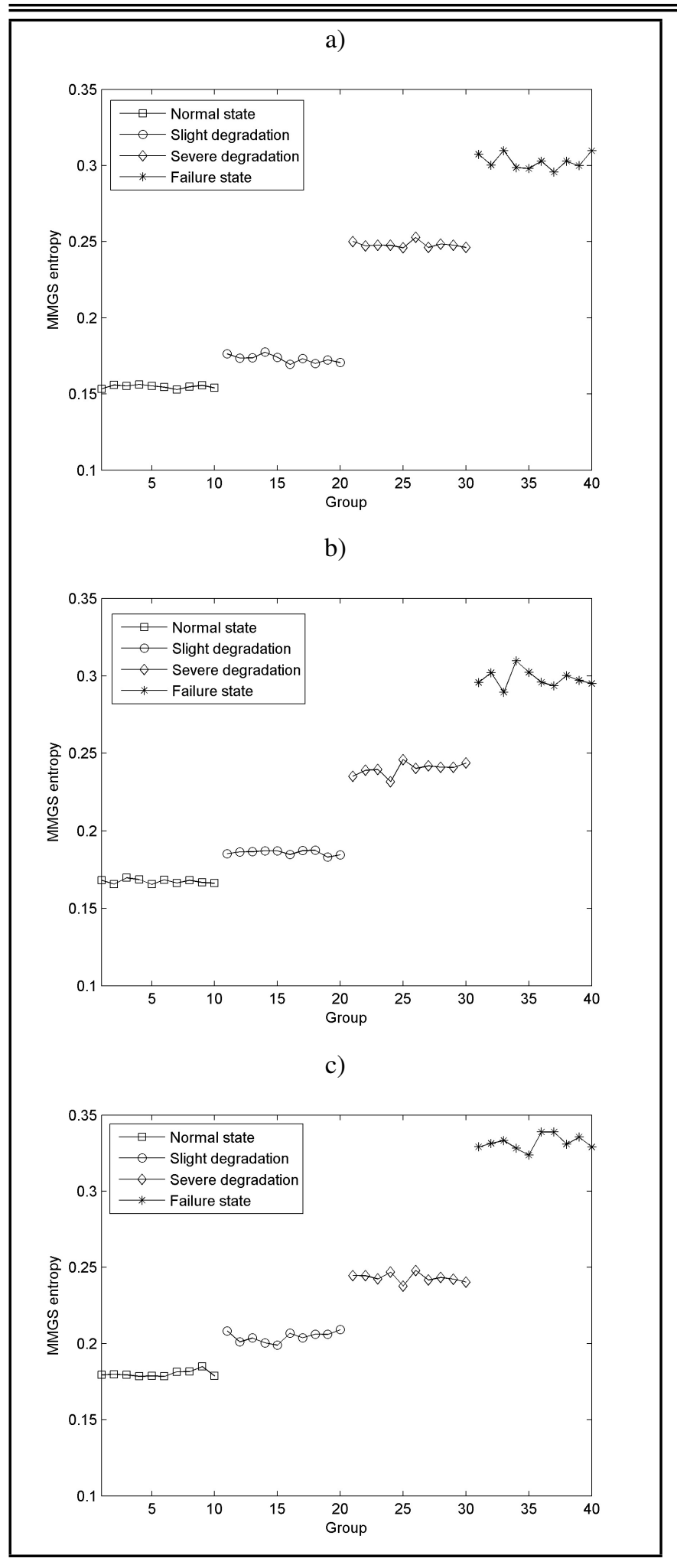

Figure 17. MMGS entropy values of training samples.

Table 2. Statistical results based on MMGS entropy.

\begin{tabular}{|c|c|c|c|c||}
\hline Load & $\begin{array}{c}\text { Test } \\
\text { sample }\end{array}$ & $\begin{array}{c}\text { Wrong } \\
\text { identification }\end{array}$ & Load & $\begin{array}{c}\text { Total } \\
\text { identification ratio }\end{array}$ \\
\hline $0 \mathrm{~N} \cdot \mathrm{m}$ & 160 & 5 & $96.88 \%$ & \\
$0.1 \mathrm{~N} \cdot \mathrm{m}$ & 160 & 9 & $94.38 \%$ & $94.79 \%$ \\
$0.2 \mathrm{~N} \cdot \mathrm{m}$ & 160 & 11 & $93.13 \%$ & \\
\hline
\end{tabular}

a)

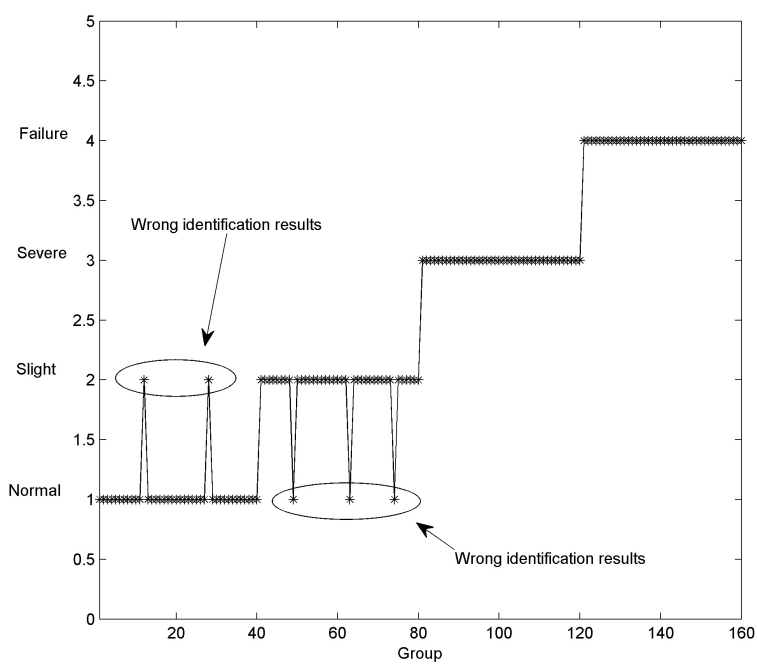

b)

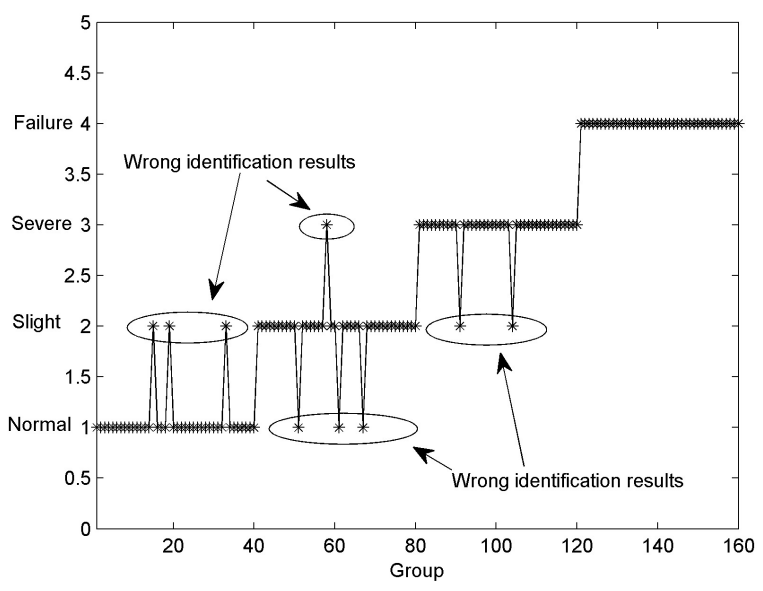

c)

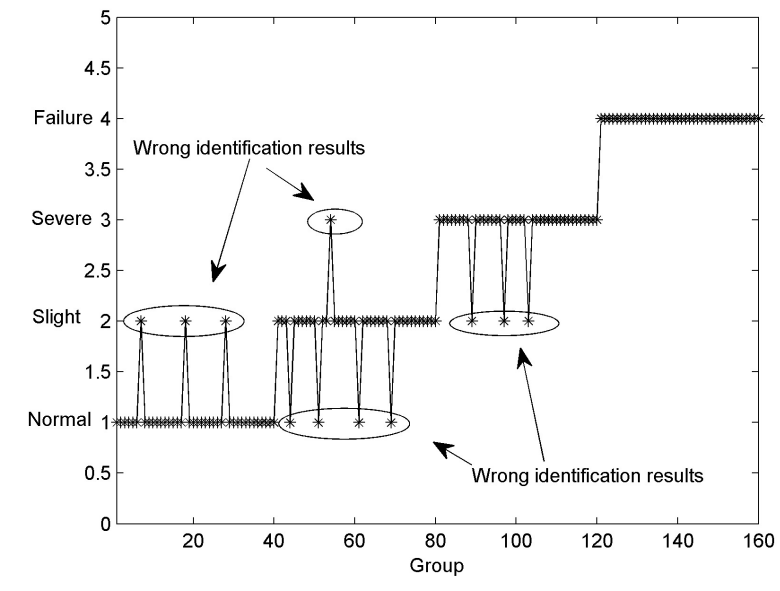

Figure 18. Degradation state identification results based on MMGS entropy.

According to the value of grey relevance between test samples and standard vector, the degradation state recognition results are shown in Fig. 18 and Tab. 2.

Compared with the method of MMGD entropy, total wrong identification between slight and severe degradation is obviously reduced, especially under $0.1 \mathrm{~N} \cdot \mathrm{m}$ and $0.2 \mathrm{~N} \cdot \mathrm{m}$ loads, 
Table 3. Statistical results based on MMGD and MMGS entropy.

\begin{tabular}{||c|c|c|c|c||}
\hline Load & $\begin{array}{c}\text { Test } \\
\text { sample }\end{array}$ & $\begin{array}{c}\text { Wrong } \\
\text { identification }\end{array}$ & Load & $\begin{array}{c}\text { Total } \\
\text { identification ratio }\end{array}$ \\
\hline $0 \mathrm{~N} \cdot \mathrm{m}$ & 160 & 2 & $98.75 \%$ & \\
$0.1 \mathrm{~N} \cdot \mathrm{m}$ & 160 & 4 & $97.5 \%$ & $97.71 \%$ \\
$0.2 \mathrm{~N} \cdot \mathrm{m}$ & 160 & 5 & $96.88 \%$ & \\
\hline
\end{tabular}

but the method based on MMGD entropy keeps the advantage in the discrimination between normal state and slight degradation.

\subsubsection{Degradation state identification based on MMGD and MMGS entropy}

In order to combining the advantages of the MMGD entropy and MMGS entropy, the constructed standard degeneration mode matrixes $E_{0}, E_{1}, E_{2}$ in three load levels are defined as follows.

$$
\begin{aligned}
& E_{0}=\left[\begin{array}{ll}
0.373 & 0.155 \\
0.428 & 0.173 \\
0.466 & 0.248 \\
0.661 & 0.302
\end{array}\right] ; \\
& E_{1}=\left[\begin{array}{ll}
0.385 & 0.167 \\
0.456 & 0.196 \\
0.481 & 0.230 \\
0.621 & 0.298
\end{array}\right] ; \\
& E_{2}=\left[\begin{array}{ll}
0.424 & 0.180 \\
0.492 & 0.204 \\
0.497 & 0.243 \\
0.661 & 0.332
\end{array}\right] .
\end{aligned}
$$

According to the value of grey relevance between test samples and standard matrixes, the degradation state recognition results are shown in Fig. 19 and Tab. 3.

It can be found that there are only 11 wrong identification results in 160 groups of test data. Total identification ratio, which benefits from the advantages of MMGD entropy and MMGS entropy, can be raised to $97.71 \%$. The analysis demonstrates the rationality and effectiveness of taking MMGD entropy and MMGS entropy as the fault feature of ceramics cracking in ultrasonic motor. Based on this, the degradation state identification method according to the gray correlation analysis is also feasible.

\section{CONCLUSIONS}

A method for fault feature extraction and degradation state identification based on multi-scale morphological gradient is proposed in this paper, which is verified by the analysis in the ultrasonic motor degradation experiment. The conclusions can be drawn as follows:

1. The cracking of piezoelectric ceramic in ultrasonic motor can affect the vibration of stator. As a result, the mathematical morphology of the voltage signal generated from a)

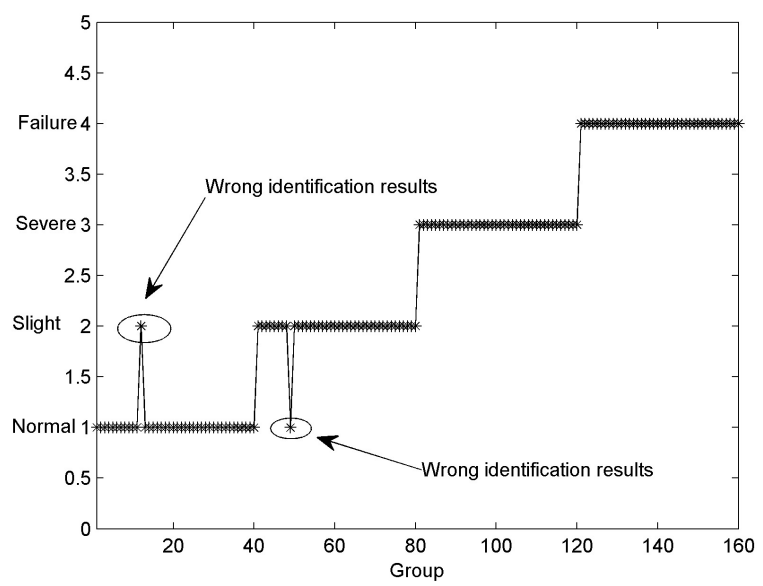

b)

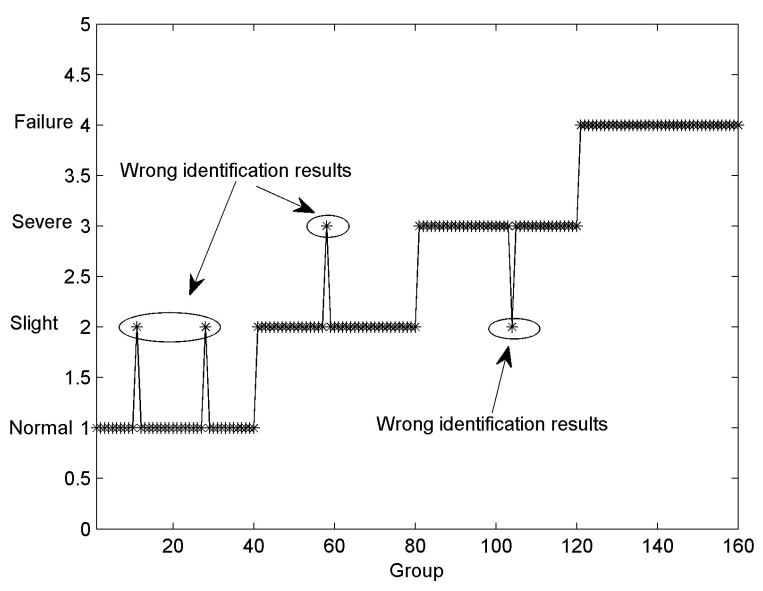

c)

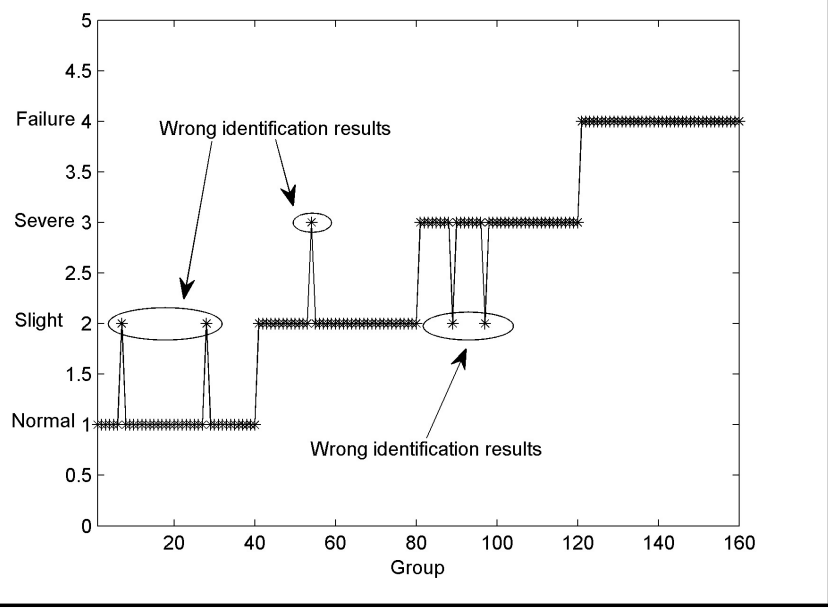

Figure 19. Degradation state identification results based on MMGD and MMGS entropy.

sensor part changes. Therefore, multi-scale morphological decomposition for the voltage signal can be used for fault feature extraction.

2. With the deterioration of ceramics, both of MMGD entropy and MMGS entropy values increase. MMGD entropy has better discrimination between normal state and slight degradation, while MMGS entropy has better anti- 
noise performance. The method based on MMGD entropy and MMGS entropy can be used to indicate the performance degradation of the piezoelectric ceramic in ultrasonic motor.

3. The proposed standard degeneration mode matrix based on MMGD entropy and MMGS entropy, can be used for grey relational analysis to improve the identification ratio. The ultrasonic motor degradation experiment shows the rationality and effectiveness of the proposed method.

\section{ACKNOWLEDGEMENTS}

This project is supported by China Postdoctoral Science Foundation (Grant No. 2017M623404), Natural Science Youth Foundation of Hebei (Grant No. E2017208086) as well as Science and Technology Research Youth Foundation for Hebei College (Grant No. QN2017329).

\section{REFERENCES}

${ }^{1}$ Stepanenko, D. A. and Minchenya, V. T. Development and Study of Novel Non-contact Ultrasonic Motor Based on Principle of Structural Asymmetry, Ultrasonics, 52 (7), 866-872, (2012). https://dx.doi.org/10.1016/j.ultras.2012.02.004

${ }^{2} \mathrm{Li}$, X., et al. Dynamic Modeling and Characteristics Analysis of a Modal-independent Linear Ultrasonic Motor, Ultrasonics, 72, 117-127, (2016). https://dx.doi.org/10.1016/j.ultras.2016.07.018

${ }^{3}$ Qu, J., et al. Characteristics of Ring Type Traveling Wave Ultrasonic Motor in Vacuum, Ultrasonics, 49 (3), 338-343, (2009). https://dx.doi.org/10.1016/j.ultras.2008.10.015

${ }^{4}$ Peng, T., et al. Experimental Investigation on Sandwich Structure Ring-type Ultrasonic Motor, Ultrasonics, 56, 303-307, (2015). https://dx.doi.org/10.1016/j.ultras.2014.08.014

${ }^{5}$ Sun, J., Hongru, L., and Zaike, T. Degradation Feature Extraction of Hydraulic Pump Based on LCDDCS Fusion Algorithm, Proceedings of the Institution of Mechanical Engineers, Part B: Journal of Engineering Manufacture, 232 (8), 1460-1470, (2016). https://dx.doi.org/10.1177/0954405416668929

${ }^{6} \mathrm{Li}$, Y., et al. Early Fault Feature Extraction of Rolling Bearing Based on ICD and Tunable QFactor Wavelet Transform, Mechanical Systems and Signal Processing, 86 (A), 204-223, (2017). https://dx.doi.org/10.1016/j.ymssp.2016.10.013

7 Pan, J., et al. Mono-component Feature Extraction for Mechanical Fault Diagnosis Using Modified Empirical Wavelet Transform via Data-driven Adap- tive Fourier Spectrum Segment, Mechanical Systems and Signal Processing, 72-73, 160-183, (2016). https://dx.doi.org/10.1016/j.ymssp.2015.10.017

${ }^{8}$ Singh, J., Darpe, A. K., and Singh, S. P. Rolling Element Bearing Fault Diagnosis Based on Overcomplete Rational Dilation Wavelet Transform and Autocorrelation of Analytic Energy Operator, Mechanical Systems and Signal Processing, 100, 662-693, (2018). https://dx.doi.org/10.1016/j.ymssp.2017.06.040

9 Wang, D., Kwok-Leung T., and Qiang Z. Novel GaussHermite Integration Based Bayesian Inference on Optimal Wavelet Parameters for Bearing Fault Diagnosis, Mechanical Systems and Signal Processing, 72-73, 80-91, (2016). https://dx.doi.org/10.1016/j.ymssp.2015.11.018

${ }^{10}$ Xue, X., et al. An Adaptively Fast Ensemble Empirical Mode Decomposition Method and Its Applications to Rolling Element Bearing Fault Diagnosis, Mechanical Systems and Signal Processing, 62-63, 444-459, (2015). https://dx.doi.org/10.1016/j.ymssp.2015.03.002

${ }^{11}$ Imaouchen, Y., et al. A Frequency-Weighted Energy Operator and Complementary Ensemble Empirical Mode Decomposition for Bearing Fault Detection, Mechanical Systems and Signal Processing, 82, 103-116, (2017). https://dx.doi.org/10.1016/j.ymssp.2016.05.009

${ }^{12}$ Lv, Y., Rui, Y., and Gangbing, S. Multivariate Empirical Mode Decomposition and Its Application to Fault Diagnosis of Rolling Bearing, Mechanical Systems and Signal Processing, 81, 219-234, (2016). https://dx.doi.org/10.1016/j.ymssp.2016.03.010

${ }_{13}$ Rai, A. and Upadhyay, S. H. Bearing Performance Degradation Assessment Based on a Combination of Empirical Mode Decomposition and K-medoids Clustering, Mechanical Systems and Signal Processing, 93, 16-29, (2017). https://dx.doi.org/10.1016/j.ymssp.2017.02.003

${ }^{14}$ Meng, L., et al. A Hybrid Fault Diagnosis Method Using Morphological Filter-Translation Invariant Wavelet and Improved Ensemble Empirical Mode Decomposition, $\mathrm{Me}$ chanical Systems and Signal Processing, 50-51, 101-115, (2015). https://dx.doi.org/10.1016/j.ymssp.2014.06.004

15 Sun, J., Hongru L., and Baohua X. The Morphological Undecimated Wavelet DecompositionDiscrete Cosine Transform Composite Spectrum Fusion Algorithm and Its Application on Hydraulic Pumps, Measurement, 94, 794-805, (2016). https://dx.doi.org/10.1016/j.measurement.2016.09.024

${ }^{16}$ Dong, Y., et al. Faults Diagnosis of Rolling Element Bearings Based on Modified Morphological Method, Mechan- 
ical Systems and Signal Processing, 25 (4), 1276-1286, (2011). https://dx.doi.org/10.1016/j.ymssp.2010.10.008

17 Zheng, Z., et al. Gear Fault Diagnosis Method Based on Local Mean Decomposition and Generalized Morphological Fractal Dimensions, Mechanism \& Machine Theory, 91, 151-167, (2015). https://dx.doi.org/10.1016/j.mechmachtheory.2015.04.009

18 Yu, D., Min, W., and Xiangmin, Ch. A Method for the Compound Fault Diagnosis of Gearboxes Based on Morphological Component Analysis, Measurement, 91, 519-531, (2016). https://dx.doi.org/10.1016/j.measurement.2016.05.087

19 Li, Y., et al. Fault Detection Method for Railway Wheel Flat Using an Adaptive Multiscale Morphological Filter, Mechanical Systems and Signal Processing, 84, 642-658, (2017). https://dx.doi.org/10.1016/j.ymssp.2016.07.009

${ }^{20}$ Li, Y., Xihui, L., and Ming, J. Z. Diagonal Slice Spectrum Assisted Optimal Scale Morphological Filter for Rolling Element Bearing Fault Diagnosis, Mechanical Systems and Signal Processing, 85, 146-161, (2017). https://dx.doi.org/10.1016/j.ymssp.2016.08.019

$21 \mathrm{Li}$, Y., et al. Train Axle Bearing Fault Detection Using a Feature Selection Scheme Based MultiScale Morphological Filter, Mechanical Systems and Signal Processing, 101, 435-448, (2018). https://dx.doi.org/10.1016/j.ymssp.2017.09.007

22 Chai, M., Zaoxiao, Z., and Quan, D. A New Qualitative Acoustic Emission Parameter Based on Shannon's Entropy for Damage Monitoring, Mechanical Systems and Signal Processing, 100, 617-629, (2017). https://dx.doi.org/10.1016/j.ymssp.2017.08.007

$23 \mathrm{He}$, D., et al. Identification of Multiple Faults in Rotating Machinery Based on Minimum Entropy Deconvolution Combined with Spectral Kurtosis, Mechanical Systems and Signal Processing, 81, 235-249, (2016). https://dx.doi.org/10.1016/j.ymssp.2016.03.016

24 Ai, Yan-Ting, et al. Fusion Information Entropy Method of Rolling Bearing Fault Diagnosis Based on Ndimensional Characteristic Parameter Distance, Mechanical Systems and Signal Processing, 88, 123-136, (2017). https://dx.doi.org/10.1016/j.ymssp.2016.11.019

25 Aouabdi, S., et al. Using Multi-scale Entropy and Principal Component Analysis to Monitor Gears Degradation via the Motor Current Signature Analysis, Mechanical Systems and Signal Processing, 90, 298-316, (2017). https://dx.doi.org/10.1016/j.ymssp.2016.12.027
26 Wang, B., Hong-ru, L., and Bao-hua, X. Motor Bearing Forecast Feature Extracting and Degradation Status Identification Based on Multi-scale Morphological Decomposition Spectral Entropy, Journal of Vibration \& Shock, 32 (22), 124-128, (2013).

$27 \mathrm{Li}$, B., et al. A Weighted Multi-scale Morphological Gradient Filter for Rolling Element Bearing Fault Detection, ISA Transactions, 50 (4), 599-608, (2011). https://dx.doi.org/10.1016/j.isatra.2011.06.003

28 Jiang, H., et al. Study on Hankel Matrix-based SVD and Its Application in Rolling Element Bearing Fault Diagnosis, Mechanical Systems and Signal Processing, 52-53 (1), 338-359, (2015). https://dx.doi.org/10.1016/j.ymssp.2014.07.019

29 Muruganatham, B., et al. Roller Element Bearing Fault Diagnosis Using Singular Spectrum Analysis, Mechanical Systems and Signal Processing, 35 (1-2), 150-166, (2013). https://dx.doi.org/10.1016/j.ymssp.2012.08.019

${ }^{30} \mathrm{Li}, \mathrm{H}$, et al. The Application of a General Mathematical Morphological Particle as a Novel Indicator for the Performance Degradation Assessment of A Bearing, Mechanical Systems and Signal Processing, 82, 490-502, (2017). https://dx.doi.org/10.1016/j.ymssp.2012.08.019

31 Ferreira, A. and Patrice M. High-performance Loadadaptive Speed Control for Ultrasonic Motors, Control Engineering Practice, 6 (1), 1-13, (1998). https://dx.doi.org/org/10.1016/S0967-0661(97)100570

$32 \mathrm{Li}$, S., et al. Temperature Evaluation of Travelingwave Ultrasonic Motor Considering Interaction Between Temperature Rise and Motor Parameters, Ultrasonics, 57 (3), 159-166, (2015). https://dx.doi.org/10.1016/j.ultras.2014.11.007

33 Jiang, H., Chen, J., Dong, G., et al. Study on Hankel matrix-based SVD and its application in rolling element bearing fault diagnosis, Mechanical Systems and Signal Processing, 52-53 (1), 338-359, (2015). https://dx.doi.org/10.1016/j.ymssp.2014.07.019

34 Muruganatham, B., Sanjith, M. A., Krishnakumar, B., et al. Roller element bearing fault diagnosis using singular spectrum analysis, Mechanical Systems and Signal Processing, 35 (1-2), 150-166, (2013). https://dx.doi.org/10.1016/j.ymssp.2012.08.019 